\title{
The Decay Rates of Traveling Waves and Spectral Analysis for a Class of Nonlocal Evolution Equations
}

\author{
G. Zhao ${ }^{1}$, S. Ruan ${ }^{2 *}$ \\ ${ }^{1}$ Department of Mathematics, Central Michigan University, Mt. Pleasant, MI 48859, USA \\ 2 Department of Mathematics, University of Miami, Coral Gables, FL 33124-4250, USA
}

\begin{abstract}
We obtain the precise decay rates of traveling waves for a class of nonlocal evolution equations arising in the theory of phase transitions and mathematical biology. We also investigate the spectrum of the operator obtained by linearizing at such a traveling wave. The detailed description of the spectrum is established.
\end{abstract}

Keywords and phrases: nonlocal evolution equation, traveling wave, exponential decay rate, spectral analysis

Mathematics Subject Classification: 35K55, 35K20, 35P05, 37L15

\section{Introduction}

In this paper, we are concerned with a class of nonlocal evolution equations of the form

$$
\frac{\partial u(x, t)}{\partial t}=d \frac{\partial^{2} u(x, t)}{\partial x^{2}}+f(u(x, t),(J * u)(x, t))
$$

for $x \in \mathbb{R}$ and $t \in \mathbb{R}^{+}$. Here $d \geq 0$ is a constant, $(J * u)(x, t):=\int_{\mathbb{R}} J(x-y) u(y, t) d y, f$ and $J$ are sufficiently smooth functions. Depending upon the constant $d$ and the nonlinearity $f$ involved, equation (1.1) may model the spatio-temporal development of various populations or epidemics (see Andreu et al. [2], Apreutesei et al. [3,5-7], Aronson [8], Bates and Zhao [13], Ruan [26], and Schumacher [27]). Similar equations have been also derived and studied from the point of view of certain continuum limits in the dynamic Ising models (see Bates and Chmaj [9], Bates and Chen [10], Bates et al. [11,12], Bates and Zhao [13], De Masi et al. [17], and references therein). Equation (1.1) has received much attention recently, the possible interest of such an equation lies in the fact that much more general types of interactions in the medium can be account for. The existence as well as the uniqueness of a traveling wave solution for reaction-diffusion equations and integro-differential equations (1.1) have been of great interest, both from a mathematical standpoint and for their applications (see Chen [15], Chen et al. [16], Huang [20], Volpert et al. [29], Weinberger [30], Zhao [31], Zhao and Ruan [32,33]). Indeed, our study of (1.1) is motivated by the following traveling wave problems.

\footnotetext{
${ }^{*}$ Corresponding author. E-mail: ruan@math.miami.edu
} 
A. Family of neurons (Chen et al. [16])

$$
\frac{\partial u}{\partial t}=-u+\int_{\mathbb{R}} J(x-y) S(u(y, t)) d y,
$$

where $m(u):=S(u)-u$ satisfies $m^{\prime}(0)<0, m^{\prime}(1)<0 . J$ is a smooth kernel satisfying

$$
J \geq 0 \text { on } \mathbb{R}, \int_{\mathbb{R}} J=1 .
$$

B. Ising model (De Masi et al. [17])

$$
\frac{\partial u}{\partial t}=\tanh \beta(J * u+h)-u,
$$

where $\beta>1$ is inverse temperature, $h$ is a constant, and $J$ is a smooth kernel supported in $[-1,1]$ satisfying (1.2).

C. Phase transition (Bates et al. $[9,11]$ )

$$
\frac{\partial u}{\partial t}=\varepsilon[J * u-u]+g(u),
$$

where $g(u)$ is a bistable function, $\lambda>0$.

D. Epidemic model (Aronson [8], Ruan [26])

$$
\frac{\partial u}{\partial t}=-\lambda u+\lambda\left\{1-\exp \left(-\frac{1}{\lambda} \int_{-\infty}^{\infty} u(y, t) k(x-y) d y\right)\right\}+\lambda i_{0}(x)
$$

where $\lambda>0$ is a constant, $i_{0}(x) \geq 0$ is a continuous function in $x \in \mathbb{R}$, and $k(y) \geq 0$ is a continuous function in $y \in \mathbb{R}$ satisfying $\int_{-\infty}^{\infty} k(y) d y=1$.

Throughout this paper, we make the following hypotheses.

(H0) $d+|c| \neq 0$.

(H1) $J \in C(\mathbb{R})$ is even, nonnegative such that

$$
\int_{\mathbb{R}} J(s) d s=1 \text { and } \int_{\mathbb{R}} J(s) e^{\rho s} d s<+\infty \quad \text { for any } \rho \in \mathbb{R} .
$$

(H2) $f \in C^{2, \alpha}(\mathbb{R} \times \mathbb{R})$ and $f(-1,-1)=f(1,1)=f(q, q)=0$, where $-1<q<1$.

(H3) $\partial_{s} f(r, s)>0$ for any fixed $(r, s) \in[-1,1] \times[-1,1]$.

(H4) $\partial_{r} f( \pm 1, \pm 1)<0$ and $\partial_{r} f( \pm 1, \pm 1)<-\partial_{s} f( \pm 1, \pm 1)$.

(H5) $\bar{f}(\cdot)=f(\cdot, \cdot)$ is bistable, i.e. $\bar{f}$ has exactly three zeros \pm 1 and $q$. There exists an interval $\left[l, l^{\prime}\right] \subset(-1,1)$ such that $q \in\left[l, l^{\prime}\right], \bar{f}^{\prime}(s) \geq 0$ for any $s \in\left[l, l^{\prime}\right]$ and $\bar{f}^{\prime}(s) \leq 0$ for any $s \in[-1,1] \backslash\left[l, l^{\prime}\right]$.

Under conditions (H1)-(H5), it is well known that equation (1.1) possesses a unique monotone traveling wave solution connecting the equilibria \pm 1 (i.e solutions of the form $u(x, t)=U(x+c t)$ for some velocity $c, \lim _{\xi \rightarrow \pm \infty} U(\xi)= \pm 1$ with $\xi=x+c t$.) However, the precise rates at which $U$ approaches the two homogeneous equilibria \pm 1 are still lacking. In this paper, we address this issue. Our main goal is to obtain the exact decay rates of traveling waves of (1.1) as $\xi \rightarrow \pm \infty$. With the right rates of convergence, we can easily establish the uniqueness of the traveling wave. Recently, a spectral analysis of traveling waves of (1.1) was made in Bates and Chen [10]. The authors considered the operator obtained by linearizing $(1.1)$ at $U$ in $C_{0}(\mathbb{R})$, the space of continuous functions which vanish at infinity. They showed that the operator has spectrum in the left half plane, bounded away from the imaginary axis except for an algebraically simple eigenvalue at zero. This fact is of crucial importance, which not only implies the exponential asymptotic stability of traveling waves but also leads to the description of dynamics of 
the codimension-one invariant stable manifolds. Here the codimension-one invariant stable manifolds are transverse to the one-dimensional manifold formed by the translates of the traveling wave. Along the same direction, much progress has been made to address the spectral stability of traveling waves of integro-differential equations (see Apreutesei et al. [4], Hupkes and Verduyn Lunel [21], and references therein). Based on our study of asymptotical behavior of traveling waves, we are able to obtain a detailed description of the spectrum of the operator in the underlying $L^{p}$ space $(1 \leq p \leq \infty)$.

The paper is organized as follows: in section 2, we investigate the exponential decay rates of traveling waves and prove their uniqueness. In section 3, we study the spectrum of the operator obtained by linearizing (1.1) at the traveling wave.

\section{Decay rates of traveling waves}

In this section, we study the asymptotical behavior of traveling waves $(c, U) \in \mathbb{R} \times C^{2}(\mathbb{R})$ which satisfy

$$
\left\{\begin{array}{l}
c U^{\prime}=d U^{\prime \prime}+f(U, J * U) \text { on } \mathbb{R} \\
\lim _{\xi \rightarrow \pm \infty} U(\xi)= \pm 1, \quad U^{\prime}>0 \text { on } \mathbb{R}
\end{array}\right.
$$

We show that the behavior of a traveling wave near $\pm \infty$ is governed by exponentials. Moreover, we determine the exact exponential decay rates of $U$ as $\xi \rightarrow \pm \infty$. For our purpose, we shall adapt the Fourier transform techniques presented in Mallet-Paret [22] and Pazy [25] (see also Bates and Zhao [13] and Zhao [31]). By differentiating equation (2.1) with respect to $\xi$, we obtain

$$
c\left(U^{\prime}\right)^{\prime}=d\left(U^{\prime}\right)^{\prime \prime}+f_{r}(U, J * U) U^{\prime}+f_{s}(U, J * U) J * U^{\prime} .
$$

From $(2.1)$

$$
\lim _{\xi \rightarrow \pm \infty} f_{r}(U, J * U)=f_{r}( \pm 1, \pm 1), \quad \lim _{\xi \rightarrow \pm \infty} f_{s}(U, J * U)=f_{s}( \pm 1, \pm 1) .
$$

Motivated by (2.2) and (2.3), we consider the linear operator $L: D(L) \subset L^{p}(\mathbb{R}, \mathbb{C}) \rightarrow L^{p}(\mathbb{R}, \mathbb{C})$ defined by

$$
L v:=d v^{\prime \prime}-c v^{\prime}+a(\xi) v+b(\xi) J * v, \quad \xi \in \mathbb{R},
$$

where $D(L):=\left\{v \in L^{p} \mid v^{\prime}, d v^{\prime \prime} \in L^{p}\right\}, a, b \in C_{b}(\mathbb{R}, \mathbb{R})$, and $1 \leq p \leq \infty$.

A special case occurs if both $a$ and $b$ are constants, we define $L_{0}: D\left(L_{0}\right) \subset L^{p}(\mathbb{R}, \mathbb{C}) \rightarrow L^{p}(\mathbb{R}, \mathbb{C})$ by

$$
L_{0} v:=d v^{\prime \prime}-c v^{\prime}+a v+b J * v, \quad \xi \in \mathbb{R},
$$

where $D\left(L_{0}\right):=D(L)$. In what follows, when convenient, $f_{r}( \pm 1, \pm 1), f_{s}( \pm 1, \pm 1)$ are denoted by $a^{ \pm}$and $b^{ \pm}$, respectively.

Let $\Delta_{0}(z): \mathbb{C} \rightarrow \mathbb{C}$ be the characteristic function associated with $L_{0}$, defined by

$$
\Delta_{0}(z)=d z^{2}-c z+a+b \int_{\mathbb{R}} J(s) e^{-z s} d s .
$$

In an attempt to solve the inhomogeneous equations

$$
L_{0} v=h, \quad h \in L^{p},
$$

we may formally take the Fourier transform to obtain

$$
\Delta_{0}(i \eta) \widehat{v}(\eta)=\widehat{h}(\eta), \quad \eta \in \mathbb{R}
$$

where $\widehat{g}(z)=(2 \pi)^{-1} \int_{\mathbb{R}} g(s) e^{-i z s} d s, \quad i=\sqrt{-1}$ and $z \in \mathbb{C}$. Note that $\Delta_{0}^{-1}(i \eta)=O\left(|\eta|^{-1}\right)$. Therefore, we can take the inverse transform of $\Delta_{0}^{-1}(i \eta)$ to obtain the solution $v$ provided $\Delta_{0}(i \eta) \neq 0$ for any $\eta \in \mathbb{R}$.

Definition 2.1. The operator $L_{0}$ is called hyperbolic if $\Delta_{0}(i \eta) \neq 0$ for any $\eta \in \mathbb{R}$. 
In what follows, for a given complex number $z \in \mathbb{C}$, we shall always denote its real part and imaginary part by $\operatorname{Re} z$ and $\operatorname{Im} z$, respectively. The following lemma ensures the existence of $\Delta_{0}^{-1}(i \eta)$ under suitable conditions.

Lemma 2.2. Suppose that $a<0, b>0$ and $b<-a$. Then

(a) The equation $\Delta_{0}(z)=0$ has precisely two real solutions $\lambda^{s}<0<\lambda^{u}$.

(b) The zeros of $\Delta_{0}(z)$ in the vertical strip $\left\{z \in \mathbb{C} \mid \lambda^{s} \leq \operatorname{Re} z \leq \lambda^{u}\right\}$ are $\lambda^{s}$ and $\lambda^{u}$. In addition, in each vertical strip $|\operatorname{Re} z| \leq K$, there lie only a finite number of zeros of $\Delta_{0}(z)$.

Proof. Set $N(z)=b \int_{\mathbb{R}} J(s) e^{-z s} d s$ and $D(z)=c z-d z^{2}-a$. Then $z$ is a zero of $\Delta_{0}(z)$ if and only if $z$ is a solution to the equation $N(z)=D(z)$. Due to the assumption, $D(0)-N(0)>0$. We now start with the case that $z$ takes on real values, note that $N(z)$ is a positive convex even function of $z$ with $\frac{\partial^{2} N}{\partial z^{2}}>0$ for any $z \neq 0$. Consequently, there exists two real roots of $N(z)=D(z)$, denoted by $\lambda^{s}$ and $\lambda^{u}$ with $\lambda^{s}<0<\lambda^{u}$. In addition, it is easy to see that $N(z)<D(z)$ if $z \in\left(\lambda^{+}, \lambda^{u}\right)$, whereas $N(z)>D(z)$ if $z \in \mathbb{R} \backslash\left(\lambda^{+}, \lambda^{u}\right)$. This confirms part $(a)$. Next let $z \in \mathbb{C}$. Note that

$$
|N(z)| \leq \int_{\mathbb{R}} J(s) e^{-\operatorname{Re} z s} d s<\operatorname{Re} D(z), \quad \forall z \in\left\{z \in \mathbb{C} \mid \lambda^{s}<\operatorname{Re} z<\lambda^{u}\right\} .
$$

Moreover, we observe that

$$
\operatorname{Re} D\left(\lambda^{s}+i \mu\right) \geq \int_{\mathbb{R}} J(t) e^{-\lambda^{s} t} d t>\int_{\mathbb{R}} J(t) e^{-\lambda^{s} t} \cos \mu t d t=\operatorname{Re} N\left(\lambda^{s}+i \mu\right)
$$

and

$$
\operatorname{Re} D\left(\lambda^{u}+i \mu\right) \geq \int_{\mathbb{R}} J(t) e^{-\lambda^{u} t} d t>\int_{\mathbb{R}} J(t) e^{-\lambda^{u} t} \cos \mu t d t=\operatorname{Re} N\left(\lambda^{u}+i \mu\right)
$$

for any $\mu \neq 0$. This yields the first part of (b). Due to the first inequality in $(2.7),|N(z)|$ is bounded in the vertical strip $|\operatorname{Re} z| \leq K, K>0$. Clearly, when restricted to such a strip, the solution set of $D(z)=N(z)$ is bounded. Since $\Delta_{0}(z)$ is an entire function over $\mathbb{C}$, there are only finitely many roots of $\Delta_{0}(z)$ in such a strip.

Lemma 2.3. Assume that the operator $L_{0}$ defined by (2.5) is hyperbolic. Then for each $1 \leq p \leq \infty$, $L_{0}: D\left(L_{0}\right) \rightarrow L^{p}$ is an isomorphism. The inverse is given by the convolution

$$
\left(L_{0}^{-1} h\right)(\xi)=\left(G_{0} * h\right)(\xi)=\int_{\mathbb{R}} G_{0}(\xi-\eta) h(\eta) d \eta
$$

with the function $G_{0}$, which enjoys the estimate

$$
\left|G_{0}(\xi)\right| \leq C e^{-\alpha|\xi|}, \quad \xi \in \mathbb{R}
$$

for some positive constants $C$ and $\alpha$. In particular, for each $h \in L^{p}, u=L_{0}^{-1} h$ is the unique solution to the inhomogeneous equation (2.6).

Proof. Set

$$
G_{0}(\xi)=\int_{\mathbb{R}} e^{i \xi \eta} \Delta_{0}^{-1}(i \eta) d \eta, \quad \xi \in \mathbb{R} .
$$

By a similar argument used in Mallet-Paret [22], we may interpret $G_{0}$ as a tempered distribution and show that

$$
d G_{0}^{\prime \prime}(\xi)-c G_{0}^{\prime}(\xi)+a G_{0}(\xi)+b J * G_{0}(\xi)=\delta(\xi),
$$

where $\delta$ denotes the delta distribution function. Therefore, when $d=0$, as a function, $G_{0}$ is absolutely continuous for all $\xi \neq 0$ and satisfies

$$
c G_{0}^{\prime}(\xi)=a G_{0}(\xi)+b J * G_{0}(\xi) \text { almost everywhere. }
$$


Furthermore, the function $G_{0}$ possesses left- and right-hand limits $G_{0}(0-)$ and $G_{0}(0+)$ at $\xi=0$, and there is a jump discontinuity

$$
G_{0}(0+)-G_{0}(0-)=1 .
$$

If $d>0$, then $G_{0}$ is absolutely continuous for all $\xi$ and $G_{0}^{\prime}$ is discontinuous at $\xi=0$.

We now show that the function $G_{0}$ decays exponentially at $\pm \infty$. First observe that

$$
\left|\int_{\mathbb{R}} J(s) e^{-z s} d s\right| \rightarrow 0, \quad|\operatorname{Im} z| \rightarrow \infty
$$

in each vertical strip $|\operatorname{Re} z| \leq K$. We then have

$$
\Delta_{0}(z)=d z^{2}-c z+O(1), \quad|\operatorname{Im} z| \rightarrow \infty
$$

uniformly in such a strip. Thanks to the assumption that $\Delta_{0}(i \eta) \neq 0$ for any $\eta \in \mathbb{R}$, Lemma 2.2 implies that there exists $\alpha>0$ such that $\Delta_{0}^{-1}(z)$ is analytic in the strip $|\operatorname{Re} z|<\alpha$. In order to obtain (2.8), we distinguish between two cases.

For the case that $d=0$, we write

$$
\Delta_{0}^{-1}(z)=[-c(z+k)]^{-1}+R(z)
$$

where $k>2 \alpha$. Clearly, in the strip $|\operatorname{Re} z|<\alpha, R(z)$ is analytic. Moreover, $R(z)$ satisfies $R(z)=$ $O(|\operatorname{Im} z|)^{-2}$ uniformly as $|\operatorname{Im} z| \rightarrow \infty$. Consequently, if $\xi \geq 0$, then we can calculate the function $G_{0}$ by shifting the path of integration in (2.9) as follows:

$$
\begin{aligned}
G(\xi) & =\frac{1}{2 \pi} \int_{\mathbb{R}} e^{i \xi s} \Delta_{0}^{-1}(i s) d s=\frac{1}{2 \pi} \int_{\mathbb{R}} e^{i \xi s}\left[-c(i s+k)^{-1}+R(i s)\right] d s \\
& =-\frac{e^{-k \xi}}{2 \pi c}+\frac{e^{-\alpha \xi}}{2 \pi} \int_{\mathbb{R}} e^{i \xi s} R(-\alpha+i s) d s .
\end{aligned}
$$

The absolute convergence of the last integral yields

$$
\left|G_{0}(\xi)\right| \leq C e^{-\alpha \xi}, \quad \xi \geq 0
$$

for some positive constant $C$. In the same manner, we can infer that

$$
\left|G_{0}(\xi)\right| \leq C e^{-\alpha \xi}, \quad \xi \leq 0 .
$$

It is evident that the same reasoning works for $d>0$ since $\Delta_{0}^{-1}(z)=O\left(|\operatorname{Im} z|^{-2}\right)$ in the strip $|\operatorname{Re} z| \leq \alpha$. Thus (2.8) is established. Furthermore, $(1+|\eta|) \Delta_{0}^{-1}(i \eta) \in L^{2}$ implies that $G \in H^{1}$ and $\widehat{G_{0}^{\prime}}=i s \Delta_{0}^{-1}(i s)$. In addition, it follows the same lines that

$$
\left|G_{0}^{\prime}(\xi)\right| \leq C e^{-\alpha|\xi|}, \quad \xi \in \mathbb{R} .
$$

We now solve the inhomogeneous problem

$$
L_{0} v=h, \quad h \in L^{p}
$$

for $h \in L^{p}$. For given $h \in L^{p}$, let $v$ be the convolution $v=G_{+} * h$. Then by Young's inequality,

$$
\|v\|_{L^{p}} \leq\left\|G_{+}\right\|_{L^{1}}\|h\|_{L^{p}} .
$$

Also note that

$$
\left\|v^{\prime}\right\|_{L^{p}} \leq\left\|G_{+}^{\prime}\right\|_{L^{1}}\|h\|_{L^{p}}
$$


provided $d \neq 0$. To verify $v$ is a solution to (2.11), it is sufficient to show that (2.11) holds everywhere for the function $v$, that is,

$$
\int_{\mathbb{R}} \chi(\xi)\left(L_{0} v\right)(\xi) d \xi=d \int_{\mathbb{R}} \chi^{\prime}(\xi) v^{\prime}(\xi) d \xi-c \int_{\mathbb{R}} \chi^{\prime}(\xi) v(\xi) d \xi+\int_{\mathbb{R}} \chi(\xi) h(\xi) d \xi
$$

for all $C^{\infty}$ functions $\chi: \mathbb{R} \rightarrow \mathbb{C}$ of compact support. Indeed, it follows from the jump condition (2.10) and Fubini's theorem that

$$
\begin{aligned}
& a \int_{\mathbb{R}} \chi(\xi)\left(G_{0} * h\right)(\xi) d \xi+b \int_{\mathbb{R}} \chi(\xi)\left(J * G_{0} * h\right)(\xi) d \xi \\
= & \left.\int_{\mathbb{R}} \chi(\xi)\left[b\left(J * G_{0}\right)+a G_{0}\right) * h\right](\xi) d \xi \\
= & \int_{\mathbb{R}}\left[\int_{\mathbb{R}} \chi(\xi)\left(c G_{0}^{\prime}(\xi-\eta)-d G_{0}^{\prime \prime}(\xi-\eta)\right) d \xi\right] h(\eta) d \eta+\int_{\mathbb{R}} \chi(\eta) h(\eta) d \eta \\
= & \int_{\mathbb{R}}\left[\int_{\mathbb{R}}\left(-c \chi^{\prime}(\xi) G_{0}(\xi-\eta)+d \chi^{\prime}(\xi) G_{0}^{\prime}(\xi-\eta)\right) d \xi\right] h(\eta) d \eta+\int_{\mathbb{R}} \chi(\eta) h(\eta) d \eta \\
= & d \int_{\mathbb{R}} \chi^{\prime}(\xi) v^{\prime}(\xi) d \xi-c \int_{\mathbb{R}} \chi^{\prime}(\xi) v(\xi) d \xi+\int_{\mathbb{R}} \chi(\xi) h(\xi) d \xi .
\end{aligned}
$$

Now, to complete the proof, we only need to show that $L_{0} u=0$ for some $u \in D\left(L_{0}\right)$ if and only if $u=0$. In fact, by interpreting $u$ as a tempered distribution and taking the Fourier transform, we have

$$
\Delta_{0}(i \eta) \widehat{u}(\eta)=0 .
$$

Since $\Delta_{0}(i \eta) \neq 0$ for any $\eta \in \mathbb{R}, \widehat{u}$ must be a zero distribution and hence $u=0$. The proof is completed

We now construct the Green's function for a small perturbation $L_{q}$ of $L_{0}$. Namely $L_{q}=L_{0}+Q$, here $Q: L^{p} \rightarrow L^{p}$ is a bounded linear operator defined by

$$
(Q v)(\xi)=m(\xi) v(\xi)+n(\xi) \int_{\mathbb{R}} J(\xi-\eta) v(\eta) d \eta,
$$

where $m, n \in L^{\infty}$.

Proposition 2.4. Let $L_{0}$ be given by (2.5) and $L_{q} v(\xi)=\left(L_{0}+Q\right) v(\xi)$. If

$$
\max \left\{\|m\|_{L^{\infty}},\|n\|_{L^{\infty}}\right\} \leq \varepsilon
$$

such that $\varepsilon$ is sufficiently small, then $L_{q}: D\left(L_{0}\right) \rightarrow L^{p}$ is an isomorphism for $1 \leq p \leq \infty$. In addition, there exist positive constants $\nu, K$, and a function $G_{q}: \mathbb{R}^{2} \rightarrow \mathbb{C}$ satisfying the pointwise estimate

$$
\left|G_{q}(\xi, \eta)\right| \leq K e^{\nu(\xi-\eta)}
$$

such that

$$
L_{q}^{-1}=\int_{\mathbb{R}} G_{q}(\xi, \eta) h(\eta) d \eta
$$

for each $h \in L^{p}$.

Proof. The proof is similar to that of Proposition 5.2 in Mallet-Paret [22], here we only provide an outline. Since $L_{0}: D\left(L_{0}\right) \rightarrow L^{p}$ is an isomorphism, $L_{q}=\left(I-Q L_{0}^{-1}\right) L_{0}$. It then follows from Theorem 1.16 of Kato [18] that

$$
L_{q}^{-1}=L_{0}^{-1} \sum_{j=0}^{\infty}\left(Q L_{0}^{-1}\right)^{j}
$$


as long as $\left\|Q L_{0}^{-1}\right\|<1$. Set

$$
\Gamma_{1}(\xi, \eta)=m(\xi) G_{0}(\xi-\eta)+n(\xi) \int_{\mathbb{R}} J(\xi-s) G_{0}(s, \eta) d s .
$$

In view of Lemma 2.3, $\left(Q L_{0}^{-1}\right)^{j}$ is an integral operator, whose kernel is defined inductively by

$$
\Gamma_{j}(\xi, \eta)=\int_{\mathbb{R}} \Gamma_{1}(\xi, \tau) \Gamma_{j-1}(\tau, \eta) d \tau
$$

for all $j \geq 2$. Thanks to (H1), a straightforward calculation shows that

$$
\left|\Gamma_{1}(\xi, \eta)\right| \leq \varepsilon C e^{-\alpha|\xi-\eta|}+\varepsilon e^{-\alpha|\xi-\eta|} \int_{\mathbb{R}} J(s) e^{\alpha|s|} d s .
$$

Therefore, there exists a positive constant $K_{1}$ such that $\left|\Gamma_{1}(\xi, \eta)\right| \leq 2 \varepsilon K_{1} e^{-\alpha|\xi-\eta|}$. In addition, by using (2.14), we infer that

$$
\left|\Gamma_{j}(\xi, \eta)\right| \leq \Psi^{* j}(\xi-\eta), \Psi(\xi)=2 \varepsilon K_{1} e^{-\nu|\xi|},
$$

where $\Psi^{* j}=\Psi * \Psi^{*(j-1)}$ is the $j$-fold convolution of $\Psi$ with itself. By Lemma 5.1 of Mallet-Paret [22], we infer that

$$
\sum_{j=1}^{\infty}\left|\Gamma_{j}(\xi, \eta)\right| \leq K e^{-\nu|\xi-\eta|}
$$

Here $\nu=\sqrt{\alpha^{2}-4 \varepsilon K_{1} \alpha}$ and $K=\frac{2 \varepsilon K_{1} \alpha}{\beta}$. Now let

$$
G_{q}(\xi, \eta)=G_{0}(\xi-\eta)+\int_{\mathbb{R}} G_{0}(\xi-s)\left(\sum_{j=1}^{\infty} \Gamma_{j}(s, \eta)\right) d s .
$$

Then a direct calculation yields

$$
\left|G_{q}(\xi, \eta)\right| \leq K e^{-\nu|\xi-\eta|}
$$

Furthermore, it is easy to see that

$$
L_{q}^{-1} h=\int_{\mathbb{R}} G_{q}(\xi, \eta) h(\eta) d \eta
$$

Therefore, the proof is completed.

Next we consider the operator $L$ defined by (2.4). Hereafter, we assume that

$$
\lim _{\xi \rightarrow+\infty} a( \pm \xi)=a^{ \pm}, \lim _{\xi \rightarrow+\infty} b( \pm \xi)=b^{ \pm},
$$

where $a^{+}, a^{-}, b^{+}$, and $b^{-}$are constants. Let $L_{ \pm}: D\left(L_{0}\right) \rightarrow L^{p}$ be the operator defined by

$$
L_{ \pm} u=d u^{\prime \prime}-c u^{\prime}+a^{ \pm}+b^{ \pm} J * u,
$$

respectively.

We also define the formally adjoint operator $L^{*}$ of $L$ to be

$$
L^{*}(\xi) v(\xi)=d v^{\prime \prime}+c v^{\prime}+a(\xi) v(\xi)+\int_{\mathbb{R}} J(\xi-\eta) b(\eta) v(\eta) d \eta .
$$

For any $\lambda \in \mathbb{C}$, it is easy to see that

$$
\int_{\mathbb{R}} \overline{v(\xi)}(L-\lambda I) u(\xi) d \xi=\int_{\mathbb{R}} \overline{\left(L^{*}-\lambda I\right) v(\xi)} u(\xi) d \xi,
$$


where $u \in W^{1, p}, v \in W^{1, q}$ and $\frac{1}{p}+\frac{1}{q}=1$.

Similarly, the formally adjoint operators $L_{ \pm}{ }^{*}$ of $L_{ \pm}$are defined by

$$
\left(L_{ \pm}^{*} v\right)(\xi)=d v^{\prime \prime}(\xi)+c v^{\prime}(\xi)+a^{ \pm} u(\xi)+b^{ \pm}(J * u)(\xi) .
$$

Given $\lambda \in \mathbb{C}$, let $\Delta_{L_{ \pm}-\lambda I}: \mathbb{C} \rightarrow \mathbb{C}$ be the characteristic functions associated with the operators $L_{ \pm}-\lambda I$, which are defined by

$$
d z^{2}-c z-\lambda+a^{ \pm}+b^{ \pm} \int_{\mathbb{R}} J(s) e^{-z s} d s .
$$

Let $\Delta_{L_{ \pm}-\bar{\lambda} I}^{*}: \mathbb{C} \rightarrow \mathbb{C}$ be the characteristic equations associated with the adjoint operators $\left(L_{ \pm}-\lambda I\right)^{*}$, which are defined by

$$
d z^{2}+c z-\bar{\lambda}+a^{ \pm}+b^{ \pm} \int_{\mathbb{R}} J(s) e^{-z s} d s
$$

Remark 2.5. In light of Lemma 2.2, it is clear that there exists $\Lambda>0$ such that $\Delta_{L_{+}-\lambda}(z)=0$ (resp. $\left.\Delta_{L_{-}-\lambda}(z)=0\right)$ has no solutions in the vertical strip $\{\lambda \in \mathbb{C} \mid-\Lambda \leq \operatorname{Re} z \leq \Lambda\}$ provided $\Delta_{L_{+}-\lambda}(i \eta) \neq 0$ (resp. $\Delta_{L_{-}-\lambda}(i \eta) \neq 0$ ) for any $\eta \in \mathbb{R}$. In fact, for any $K>0$, the set $\left\{-K \leq \operatorname{Re} z \leq K \mid \Delta_{L_{ \pm}-\lambda}(z)=0\right\}$ is bounded. Since $\Delta_{L_{ \pm}-\lambda}(z)$ is analytic on $\mathbb{C}$, there are only a finite number of zeros of $\Delta_{L_{ \pm}-\lambda}(z)$ located in the strip $-K \leq \operatorname{Re} z \leq K$, thus, there must exist $\Lambda>0$ such that $\Delta_{L_{ \pm}-\lambda}(z)=0$ has no solutions in the vertical strip $\{\lambda \in \mathbb{C} \mid-\Lambda \leq \operatorname{Re} z \leq \Lambda\}$. In addition, if $\Delta_{L_{+}-\lambda I}(i \eta) \neq 0$ (resp. $\Delta_{L_{-}-\lambda I}(i \eta) \neq 0$ ) for any $\eta \in \mathbb{R}$, then $\Delta_{L_{+}-\bar{\lambda} I}^{*}(i \eta) \neq 0$ (resp. $\Delta_{L_{-}-\bar{\lambda} I}^{*}(i \eta) \neq 0$ ) for any $\eta \in \mathbb{R}$.

Definition 2.6. The operator $L_{ \pm}-\lambda I$ are called hyperbolic if $\Delta_{L_{ \pm}-\lambda I}(i \eta) \neq 0$ for any $\eta \in \mathbb{R}$. The operator $L-\lambda I$ is called asymptotically hyperbolic if both $L_{+}-\lambda I$ and $L_{-}-\lambda I$ are hyperbolic. Similarly, the operator $\left(L_{ \pm}-\lambda I\right)^{*}$ are called hyperbolic if $\Delta_{L_{ \pm}-\lambda I}^{*}(i \eta) \neq 0$ for any $\eta \in \mathbb{R}$. The operator $(L-\lambda I)^{*}$ is called asymptotically hyperbolic if both $\left(L_{+}-\lambda I\right)^{*}$ and $\left(L_{-}-\lambda I\right)^{*}$ are hyperbolic.

Proposition 2.7. If $\lambda \in \mathbb{C}$ such that $L_{+}-\lambda I$ is hyperbolic, then the operator $L_{+}-\lambda I$ is an isomorphism from $W^{1, p}$ onto $L^{p}$ for $1 \leq p \leq \infty$ provided $d=0$. If $d>0$, then $L_{+}-\lambda I$ is an isomorphism from $W^{2, p}$ onto $L^{p}$ for $1 \leq p \leq \infty$. In each case, the inverse is given by the convolution

$$
\left[\left(L_{+}-\lambda I\right)^{-1} h\right](\xi)=\left(G_{+}^{\lambda} * h\right)(\xi)=\int_{\mathbb{R}} G_{+}^{\lambda}(\xi-\eta) h(\eta) d \eta
$$

with a function $G_{+}^{\lambda}$ which enjoys the estimate

$$
\left|G_{+}^{\lambda}(\xi)\right| \leq K^{\prime} e^{-\alpha|\xi|}, \quad \xi \in \mathbb{R}
$$

for some constants $K^{\prime}$ and $\alpha$. Moreover, the same assertion is valid for $L_{-}-\lambda I$.

Proof. Invoking Lemma 2.3, we only need to show (2.19). By the Remark 2.5, there exist $m>0$ such that all zeros of $\Delta_{L_{+-\lambda}}$ lie outside of the strip $\{\lambda \in \mathbb{C}|| \operatorname{Re} z \mid \leq m\}$. We define

$$
k^{+}=\inf \left\{\operatorname{Re} z: \Delta_{L_{+}-\lambda}(z)=0, \operatorname{Re} z>0\right\}
$$

and

$$
k^{-}=\sup \left\{\operatorname{Re} z: \Delta_{L_{+}-\lambda}(z)=0, \operatorname{Re} z<0\right\} .
$$

Choose $\varepsilon^{\prime}>0$ sufficiently small such that $\Delta_{L_{+}-\lambda}(z)$ only has a finite number of zeros in the strip $k_{-}-\varepsilon^{\prime}<\operatorname{Re} z \leq \varepsilon^{\prime}$ and $\Delta_{L_{+}-\lambda}(z)$ is analytic on $\operatorname{Re} z=k_{-}-\varepsilon^{\prime}$. Again, we let

$$
G_{+}^{\lambda}(\xi)=\int_{\mathbb{R}} e^{i \xi \eta} \Delta_{L_{+}-\lambda}^{-1}(i \eta) d \eta
$$


By the reasoning used in the proof of Lemma 2.3, we find, for any $\xi \geq 0$, that

$$
\begin{aligned}
G_{+}^{\lambda}(\xi) & =\left.\sum \operatorname{Res}\left(e^{z \xi} \Delta_{L_{+}-\lambda}^{-1}(z)\right)\right|_{k_{-}-\varepsilon^{\prime}<\operatorname{Re} z \leq \varepsilon^{\prime}}+e^{\left(k_{-}-\varepsilon^{\prime}\right) \xi} \int_{\mathbb{R}} e^{i \xi \eta} \Delta_{L_{+}-\lambda}^{-1}\left(k^{-}-\varepsilon^{\prime}+i \eta\right) d \eta \\
& =O\left(\xi^{i} e^{k_{-} \xi}\right) \text { as } \xi \rightarrow \infty .
\end{aligned}
$$

Here we assume that the zero with $\operatorname{Re} z=k_{-}$is the $i$ th zero of $\Delta_{L_{+}-\lambda}(z)$ in the strip $k^{-}-\varepsilon^{\prime}<\operatorname{Re} z \leq \varepsilon^{\prime}$. Analogously, we have

$$
G_{+}^{\lambda}(\xi)=O\left(\xi^{j} e^{k_{+} \xi}\right) \text { as } \xi \rightarrow-\infty
$$

for some $j \geq 0$. Let $0<\alpha<\min \left\{\left|k_{-}\right|,\left|k_{+}\right|\right\}$, then (2.19) follows.

Lemma 2.8. Assume that $\lambda \in \mathbb{C}$ such that $L-\lambda I$ is asymptotically hyperbolic. Suppose $(L-\lambda I) u=h$, where $h \in L^{p} . u \in W^{1, p}$ when $d=0$, and $u \in W^{2, p}$ when $d \neq 0$. Then

$$
\begin{gathered}
|u(\xi)| \leq K_{1} e^{-\mu|\xi|}\|u\|_{L^{\infty}}+K_{2} \int_{\mathbb{R}} e^{-\mu|\xi-\eta|}|h(\eta)| d \eta, \quad \xi \in \mathbb{R} . \\
\|u\|_{W^{1, p}} \leq K_{3}\|u\|_{L^{p}}+K_{4}\|h\|_{L^{p}} \quad \text { if } d=0 . \\
\|u\|_{W^{2, p}} \leq K_{5}\|u\|_{L^{p}}+K_{6}\|h\|_{L^{p}} \quad \text { if } d>0 .
\end{gathered}
$$

Here all the constants $\mu$ and $K_{i}(i=1, \cdot, \cdot, 6)$ are positive and independent of $u$ and $h$.

Proof. Let $G_{+}^{\lambda}$ and $G_{-}^{\lambda}$ be the Green functions for $L_{+}-\lambda I$ and $L_{-}-\lambda I$, respectively. Invoking Proposition 2.7, we may assume that both $G_{+}^{\lambda}$ and $G_{-}^{\lambda}$ enjoy the estimate (2.19). Due to the assumption, for any $\varepsilon>0$, there exists $\tau(\varepsilon)>0$ such that $\left|a( \pm \xi)-a^{ \pm}\right| \leq \varepsilon$ and $\left|b( \pm \xi)-b^{ \pm}\right| \leq \varepsilon$ whenever $\xi \geq \tau$. Now let

$$
\theta_{\tau}(\xi)= \begin{cases}1, & \xi \geq \tau \\ 0, & \xi<\tau\end{cases}
$$

Let the bounded linear operators $Q^{ \pm}: L^{p} \rightarrow L^{p}$ be defined by

$$
\left(Q^{ \pm} v\right)(\xi)=\theta_{\tau}( \pm \xi)\left[a(\xi)-a^{ \pm}\right]+\theta_{\tau}(\xi)\left[b(\xi)-b^{ \pm}\right] \int_{\mathbb{R}} J(\xi-\eta) v(\eta) d \eta
$$

respectively. Set $L_{q^{ \pm}}=L_{ \pm}+Q^{ \pm}$. It is clear that $L_{q^{ \pm}} u_{n}=\left(L_{q^{ \pm}}-L\right) u_{n}+f_{n}$. Choose $\tau$ such that $Q_{ \pm}$ satisfy the conditions of Proposition 2.4, then $L_{q^{ \pm}}-\lambda I$ are isomorphisms from $W^{1, p}\left(W^{2, p}\right)$ onto $L^{p}$ for $1 \leq p \leq \infty$. Let $G_{q^{ \pm}}^{\lambda}$ be the Green functions for $L_{q^{ \pm}}$, respectively, then Proposition 2.4 yields

$$
\left|G_{q^{ \pm}}^{\lambda}(\xi, \eta)\right| \leq C_{\lambda} e^{-\mu|\xi-\eta|}
$$

for some positive constants $C_{\lambda}$ and $\mu$. Consequently, we have either

$$
\begin{aligned}
u(\xi) & =\int_{\mathbb{R}} G_{q^{+}}^{\lambda}(\xi, \eta)\left[L_{q^{+}}-L\right] u(\eta) d \eta+\int_{\mathbb{R}} G_{q^{+}}^{\lambda}(\xi, \eta) h(\eta) d \eta \\
& =\int_{-\infty}^{\tau} G_{q^{+}}^{\lambda}(\xi, \eta)\left[L_{q^{+}}-L\right] u(\eta) d \eta+\int_{\mathbb{R}} G_{q^{+}}^{\lambda}(\xi, \eta) h(\eta) d \eta \\
& \leq \int_{-\infty}^{\tau} e^{-\mu|\xi-\eta|}\left\|L_{q^{+}}-L\right\|\|u\|_{L^{\infty}} d \eta+\int_{-\infty}^{\infty} e^{-\mu|\xi-\eta|}|h(\eta)| d \eta
\end{aligned}
$$

or

$$
\begin{aligned}
u(\xi) & =\int_{\mathbb{R}} G_{q^{-}}^{\lambda}(\xi, \eta)\left[L_{q^{-}}-L\right] u(\eta) d \eta+\int_{\mathbb{R}} G_{q^{-}}^{\lambda}(\xi, \eta) h(\eta) d \eta \\
& \leq \int_{\tau}^{\infty} e^{-\mu|\xi-\eta|}\left\|L_{q^{-}}-L\right\|\|u\|_{L^{\infty}} d \eta+\int_{-\infty}^{\infty} e^{-\mu|\xi-\eta|}|h(\eta)| d \eta
\end{aligned}
$$


Thus, (2.20) follows. Now we define

$$
\left(\Lambda_{\omega} v\right)(\xi)=d v^{\prime \prime}(\xi)-c v^{\prime}(\xi)-\omega v(\xi)
$$

then

$$
\Lambda_{\omega} u=(\lambda-\omega) u-P u+h,
$$

where $(P v)(\xi):=a(\xi) v(\xi)+b(\xi) J * v(\xi)$. As long as $\omega>0$ is sufficiently large, $\Lambda_{\omega}^{-1}: L^{p} \rightarrow W^{1, p}\left(W^{2, p}\right)$ exists and satisfies $\left\|\Lambda_{\omega}^{-1} u\right\|_{W^{1, p}} \leq \omega_{p}\|u\|_{L^{p}}$ provided $d=0\left(\left\|\Lambda_{\omega}^{-1} u\right\|_{W^{1, p}} \leq \omega_{p}\|u\|_{L^{p}}\right.$ if $\left.d>0\right)$, where $\omega_{p}$ only depends on $d, c, \omega$ and $p$. Since $P$ is a bounded operator in $L^{p}$, the desired conclusions (2.21) and (2.22) follow.

Remark 2.9. In virtue of Remark 2.5 and Lemma $2.8,(L-\lambda I)^{*}$ is asymptotically hyperbolic if and only if $L-\lambda I$ is asymptotically hyperbolic, where $\lambda \in \mathbb{C}$. Suppose that $L-\lambda I$ is asymptotically hyperbolic and $\mathcal{N}(L-\lambda I)$ is nonempty, where $\mathcal{N}(L-\lambda I)$ is the kernel of $L-\lambda I$. Let $\phi \in \mathcal{N}(L-\lambda I)$, then Lemma 2.8 implies that $\phi$ decays exponentially at infinity. Clearly, the same conclusion holds for $(L-\lambda I)^{*}$ provided it has a nonempty kernel.

Proposition 2.10. Assume that (2.16) is satisfied and $L-\lambda I$ is asymptotically hyperbolic for some $\lambda \in \mathbb{C}$. Suppose that there are bounded sequences $\left\{u_{n}\right\} \in D(L)$ and $\left\{h_{n}\right\} \in L^{p}$ such that $(L-\lambda I) u_{n}=h_{n}$ and $h_{n} \rightarrow h^{*}$ in $L^{p}$. Then there exist a subsequence $\left\{u_{n^{\prime}}\right\}$ and some $u^{*} \in D(L)$ such that $u_{n^{\prime}} \rightarrow u^{*}$ in $D(L)$ and $(L-\lambda I) u^{*}=h^{*}$. The same conclusion holds for $L^{*}$.

Proof. Due to the assumption and Lemma 2.8, the embedding theorem implies that the sequence $\left\{u_{n}\right\}$ is equicontinuous on any compact interval. In case of $d>0,\left\{u_{n^{\prime}}\right\}$ is also equicontinuous on any compact interval. When $p=1$, by means of an argument similar to the one used in Mallet-Paret [22], it can be shown that the above conclusions are still true. Therefore there is a subsequence, still labeled by $\left\{u_{n}\right\}$, which converges to $u^{*}$ uniformly on any compact interval for some function $u^{*}: \mathbb{R} \rightarrow \mathbb{C}$. Clearly, $u^{*} \in L^{\infty}$ since $\left\{u_{n}\right\}$ is bounded in $W^{1, p}$.

Next we show that $u_{n} \rightarrow u^{*}$ in $L^{p}$ if $1 \leq p<\infty$. By the assumption, there exists $\widehat{C}>0$ such that $\left\|u_{n}\right\|_{L^{\infty}} \leq \widehat{C}$. Let

$$
g_{n}(\xi)=K_{2} \int_{\mathbb{R}} e^{-\mu|\xi-\eta|}\left|h_{n}(\eta)\right| d \eta, \quad g^{*}(\xi)=K_{2} \int_{\mathbb{R}} e^{-\mu|\xi-\eta|}\left|h^{*}(\eta)\right| d \eta,
$$

then it follows from $(2.20)$ that

$$
\left|u_{n}(\xi)\right| \leq \widehat{C} K_{1} e^{-\mu|\xi|}+g_{n}(\xi), \quad \xi \in \mathbb{R} .
$$

By using the Hölder inequality and Young inequality, we find that $g_{n}, g^{*} \in L^{p} \cap L^{\infty}$, and

$$
\lim _{n \rightarrow \infty}\left\|g_{n}-g^{*}\right\|_{L^{\infty}}=0, \quad \lim _{n \rightarrow \infty}\left\|g_{n}-g^{*}\right\|_{L^{p}}=0 .
$$

The generalized Lebesgue dominated convergence theorem implies $\left\|u_{n}\right\|_{L^{p}} \rightarrow\left\|u^{*}\right\|_{L^{p}}$. Finally, Brézis-Lieb Lemma (Brezis [14]) yields

$$
\lim _{n \rightarrow \infty}\left\|u_{n}-u^{*}\right\|_{L^{p}}=0
$$

Due to $(2.24)$, we have

$$
u_{n}=\Lambda_{\omega}^{-1}\left((\lambda-\omega) I u_{n}-P u_{n}+h_{n}\right) .
$$

By passing the limit $n \rightarrow \infty$, we see that

$$
u^{*}=\Lambda_{\omega}^{-1}\left((\lambda-\omega) I u^{*}-P u^{*}+h^{*}\right) .
$$

Namely,

$$
(L-\lambda I) u^{*}=h^{*} .
$$


Furthermore, applying (2.21) or (2.22) to the difference $u_{n}-u^{*}$ yields

$$
u_{n} \rightarrow u^{*} \text { in } W^{1, p}\left(\text { or } W^{2, p} \text { if } d>0\right) .
$$

It remains to show that the assertion is valid when $p=\infty$. We first write for each $u_{n}$ in the form

$$
c u_{n}\left(\xi_{1}\right)=c u_{n}\left(\xi_{2}\right)+\int_{\xi_{2}}^{\xi_{1}}\left[\left(P u_{n}\right)(\eta)-h_{n}(\eta)\right] d \eta \text { for } d=0
$$

and

$$
d u_{n}^{\prime}\left(\xi_{1}\right)=d u_{n}^{\prime}\left(\xi_{2}\right)+\int_{\xi_{2}}^{\xi_{1}}\left[c u_{n}^{\prime}(\eta)-\left(P u_{n}\right)(\eta)+h_{n}(\eta)\right] d \eta \text { for } d>0,
$$

where $-\infty<\xi_{2}<\xi_{1}<\infty$. Since $\left(J * u_{n}\right)(\cdot)$ converges to $\left(J * u^{*}\right)(\cdot)$ pointwise and $J * u_{n}$ is uniformly bounded, upon taking the limit, we find

$$
c u^{*}\left(\xi_{1}\right)=c u^{*}\left(\xi_{2}\right)+\int_{\xi_{2}}^{\xi_{1}}\left[\left(P u^{*}\right)(\eta)-h_{n}(\eta)\right] d \eta \text { for } d=0
$$

and

$$
d u^{* \prime}\left(\xi_{1}\right)=d u^{* \prime}\left(\xi_{2}\right)+\int_{\xi_{2}}^{\xi_{1}}\left[c u^{* \prime}(\eta)-\left(P u^{*}\right)(\eta)+h_{n}(\eta)\right] d \eta \text { for } d>0 .
$$

Therefore, we have

$$
d\left(u^{*}\right)^{\prime \prime}(\xi)-c\left(u^{*}\right)^{\prime}(\xi)+\left(P u^{*}\right)(\xi)=h(\xi) \text { for any } \xi \in \mathbb{R} .
$$

Applying (2.20) to $u_{n}-u^{*}$ yields that

$$
\left|\left(u_{n}-u^{*}\right)(\xi)\right| \leq 2 \widehat{C} K_{1} e^{-\mu|\xi|} d \xi+K_{2} \mu\left\|h_{n}-h^{*}\right\|_{L^{\infty}}
$$

Since $h_{n} \rightarrow h^{*}$ in $L^{\infty}$, for any $\varepsilon>0$, there exist positive constants $N(\varepsilon)$ and $T(\varepsilon)$ such that $\left|\left(u_{n}-u^{*}\right)(\xi)\right| \leq$ $\frac{1}{2} \varepsilon$ whenever $n>N(\varepsilon)$ and $|\xi|>T(\varepsilon)$. In addition, we already know that $\left\{u_{n}\right\}$ converges uniformly to $u^{*}$ on any compact interval. Hence, there exists $\widetilde{N}(\varepsilon)>0$ such that $\left\|\left(u_{n}-u^{*}\right)\right\|_{L^{\infty}} \leq \varepsilon$ if $n>\widetilde{N}(\varepsilon)$. Once again, the similar reasoning shows that $u_{n} \rightarrow u^{*}$ in $W^{1, \infty}$ (or $W^{2, \infty}$ ). Thus, the proof is completed.

Proposition 2.11. Assume that $\lambda \in \mathbb{C}$ such that $(L-\lambda I)^{*}$ is asymptotically hyperbolic. Suppose for some $p$ that there are bounded sequences $\left\{u_{n}\right\} \in W^{1, p}\left(W^{2, p}\right.$ when $\left.d>0\right)$ and $\left\{h_{n}\right\} \in L^{p}$ such that $(L-\lambda I)^{*} u_{n}=h_{n}$ and $h_{n} \rightarrow \bar{h}$ in $L^{p}$. Then there exists a subsequence $\left\{u_{n^{\prime}}\right\}$ and some $\bar{u} \in W^{1, p}\left(W^{2, p}\right)$ such that $u_{n^{\prime}} \rightarrow \bar{u}$ in $W^{1, p}\left(W^{2, p}\right)$ and $(L-\lambda I)^{*} \bar{u}=\bar{h}$.

Proof. The proof is almost the same as Lemma 2.10, and hence shall be omitted.

Lemma 2.12. Suppose that $L-\lambda I$ is asymptotically hyperbolic. Then the operator $L-\lambda I: L^{p} \rightarrow L^{p}$ is a Fredholm operator for each $p(1 \leq p<\infty)$. Furthermore, the range $\mathcal{R}(L-\lambda I)$ is given by

$$
\left.\mathcal{R}(L-\lambda I)=\left\{h \in L^{p} \mid \int_{\mathbb{R}} \bar{w}(\xi) h(\xi) d \xi=0, \quad w(\xi) \in \mathcal{N}(L-\lambda I)^{*}\right)\right\} .
$$

In particular,

$$
\begin{gathered}
\operatorname{dim} \mathcal{N}(L-\lambda I)^{*}=\operatorname{codim} \mathcal{R}(L-\lambda I), \quad \operatorname{dim} \mathcal{N}(L-\lambda I)=\operatorname{codim} \mathcal{R}(L-\lambda I)^{*}, \\
\operatorname{Ind}(L-\lambda I)=-\operatorname{Ind}(L-\lambda I)^{*} .
\end{gathered}
$$

In case $p=\infty, L-\lambda I$ is a semi-Fredholm operator. Additionally, the operator $L-\lambda I$ is Fredholm if $J$ has compact support. 
Proof. The proof of this lemma is very similar to that of Theorem A in Mallet-Paret [22], we shall therefore only sketch the proof. As usual, we shall only give the proof for the case that $d=0$ since the proof for the case that $d>0$ can be completed similarly. We start to show that the unit ball

$$
\mathcal{B}=\left\{u \in W^{1, p} \mid u \in \mathcal{N}(L-\lambda I),\|u\|_{W^{1, p}} \leq 1\right\}
$$

in $\mathcal{N}(L-\lambda I) \subset W^{1, p}$ is compact, and hence we can conclude $\operatorname{dim} \mathcal{N}(L-\lambda I)<\infty$. It is worth pointing out that $\mathcal{N}(L-\lambda I)$ is independent of $p$. Indeed, this can be inferred from Remark 2.9. Now, we choose any sequence $\left\{u_{n}\right\} \in \mathcal{B}$, then by Proposition 2.10 with $h_{n}=0$, there exists a subsequence $\left\{u_{n^{\prime}}\right\}$ such that $u_{n^{\prime}} \rightarrow u^{*}$ in $W^{1, p}$ for some $u^{*}$ with $(L-\lambda I) u^{*}=0$. Therefore, $u^{*} \in \mathcal{B}$ and $\mathcal{B}$ is compact.

Next we let $p$ be fixed and we show that $\mathcal{R}(L-\lambda I)$ is closed. Let $\left\{h_{n}\right\} \in \mathcal{R}(L-\lambda I) \subseteq L^{p}$ such that $h_{n} \rightarrow h^{*}$ in $L^{p}$, then we need to show that $h^{*} \in \mathcal{R}(L-\lambda I)$. Let $\mathcal{C} \subseteq W^{1, p}$ be a closed subspace complement of $\mathcal{N}(L-\lambda I)$, that is, $W^{1, p}=\mathcal{N}(L-\lambda I) \oplus \mathcal{C}$. Clearly, there exists a sequence $\left\{u_{n}\right\} \in \mathcal{C}$ such that $(L-\lambda I) u_{n}=h_{n}$. As shown in Mallet-Paret [22], $\left\|u_{n}\right\|_{W^{1, p}}$ must be bounded, hence Proposition 2.10 implies that there exists $u^{*} \in \mathcal{C}$ such that $(L-\lambda I) u^{*}=h^{*}$. This proves the closeness of $\mathcal{R}(L-\lambda I)$. Therefore, $L-\lambda I$ is semi-Fredholm.

Now, we assume that $1 \leq p<\infty$, in order to prove that $(L-\lambda I)$ is Fredholm, it suffices to show that $\mathcal{R}(L-\lambda I)$ has a finite codimension in $L^{p}$. To this end, we let $\left(\mathcal{N}(L-\lambda I)^{*}\right)_{p}^{0} \subseteq L^{p}$ denote

$$
\left(\mathcal{N}(L-\lambda I)^{*}\right)_{p}^{0}=\left\{h \in L^{p} \mid \int_{\mathbb{R}} \bar{w}(\xi) h(\xi) d \xi=0, \quad w(\xi) \in \mathcal{N}\left((L-\lambda I)^{*}\right)\right\} .
$$

From Remark 2.9, we see that $(L-\lambda I)^{*}$ is also asymptotically hyperbolic, and hence Proposition 2.10 together with the above arguments imply that $\operatorname{dim} \mathcal{N}\left((L-\lambda I)^{*}\right)<\infty$, where $\left.\mathcal{N}(L-\lambda I)^{*}\right) \subseteq W^{1, q}$. Certainly, $\left.\operatorname{codim}\left(\mathcal{N}(L-\lambda I)^{*}\right)_{p}^{0}=\operatorname{dim} \mathcal{N}(L-\lambda I)^{*}\right)<\infty$. To complete the proof, we show that $(\mathcal{N}(L-$ $\left.\lambda I)^{*}\right)_{p}^{0}=\mathcal{R}(L-\lambda I)$. In view of $(2.17)$, we have $\mathcal{R}(L-\lambda I) \subseteq\left(\mathcal{N}(L-\lambda I)^{*}\right)_{p}^{0}$. Assume for some $p$ $(1 \leq p<\infty)$ that $\mathcal{R}(L-\lambda I) \neq\left(\mathcal{N}(L-\lambda I)^{*}\right)_{p}^{0}$, then there exists $v^{*} \in \mathcal{R}(L-\lambda I)^{\perp}$ and $\int_{\mathbb{R}} \overline{v^{*}(\xi)} h(\xi) d \xi \neq 0$ for some $h \in\left(\mathcal{N}(L-\lambda I)^{*}\right)_{p}^{0}$, where $\mathcal{R}(L-\lambda I)^{\perp}=\left\{v \in L^{q} \mid \int_{\mathbb{R}} \overline{v(\xi)} g(\xi)=0, \quad g \in \mathcal{R}(L-\lambda I)\right\}$. Clearly, $v^{*} \notin \mathcal{N}\left((L-\lambda I)^{*}\right)$. On the other hand, we have

$$
\int_{\mathbb{R}} \overline{v(\xi)}(L-\lambda I) u(\xi) d \xi=0, \quad u \in W^{1, p} .
$$

Choose any $\chi \in C^{\infty}(\mathbb{R}, \mathbb{C})$ with compact support and set $u=\bar{\chi}$. By taking the complex conjugates, we find

$$
\begin{aligned}
0 & =\int_{\mathbb{R}} v(\xi) \overline{(L-\lambda I) \overline{\chi(\xi)}} d \xi \\
& =d \int_{\mathbb{R}} \chi(\xi) v^{\prime \prime}(\xi) d \xi+c \int_{\mathbb{R}} \chi(\xi) v^{\prime}(\xi) d \xi+\int_{\mathbb{R}} \chi(\xi)\left[(a(\xi)-\bar{\lambda}) v(\xi)+\int_{\mathbb{R}} J(\xi-\eta) b(\eta) v(\eta) d \eta\right] d \xi \\
& =\int_{\mathbb{R}} \chi(\xi)\left((L-\lambda I)^{*} v\right)(\xi) d \xi .
\end{aligned}
$$

This indicates that $v$ solves the adjoint equation in the sense of distributions and $v \in W^{1, q}$. Thus $v \in \mathcal{N}(L-\lambda I)^{*}$. This contradiction establishes that $\mathcal{R}(L-\lambda I)=\left(\mathcal{N}(L-\lambda I)^{*}\right)_{p}^{0}$ for $1 \leq p<\infty$.

For the case of $p=\infty$, once again, (2.17) implies that $\mathcal{R}(L-\lambda I) \subseteq\left(\mathcal{N}(L-\lambda I)^{*}\right)_{\infty}^{0}$. Suppose that $J$ has compact support. We need to show that $\mathcal{R}(L-\lambda I) \supseteq\left(\mathcal{N}(L-\lambda I)^{*}\right)_{\infty}^{0}$. We start to show that every $h \in\left(\mathcal{N}(L-\lambda I)^{*}\right)_{\infty}^{0}$ can be written as $h=h_{1}+h_{2}$, where $h_{1} \in \mathcal{R}(L-\lambda I)$, and $h_{1}=h$ whenever $|\xi| \geq \tau$ for some positive constant $\tau$. Certainly, $h_{2} \in\left(\mathcal{N}(L-\lambda I)^{*}\right)_{\infty}^{0}$ and $h_{2}$ has compact support. Therefore, $h_{2} \in\left(\mathcal{N}(L-\lambda I)^{*}\right)_{p}^{0}$. As shown before, $h_{2} \in \mathcal{R}(L-\lambda I)$. Hence we have $h \in \mathcal{R}(L-\lambda I)$ as desired. In view of Lemma 2.8, $L_{q^{ \pm}}-\lambda I$ are isomorphisms given in Lemma 2.8 when $\tau$ is sufficiently large. To construct $h_{1}$, we let $w_{ \pm}=\left(L_{q^{ \pm}}-\lambda I\right)^{-1} h$. Namely, $(L-\lambda I) w_{+}=h$ for any $\xi \geq \tau$, while $(L-\lambda I) w_{-}=h$ for any 
$\xi \leq-\tau$. Now, we let $w^{*}(\xi)=m(\xi) w_{+}(\xi)+(1-m(\xi)) w_{-}(\xi)$, where $m: \mathbb{R} \rightarrow \mathbb{R}^{+}$is a $C^{2}$ function such that $m(\xi)=0$ for $\xi \leq 0$, and $m(\xi)=1$ for $\xi \geq 1$. Since $J$ has compact support, the direct computation shows that $(L-\lambda I) w^{*}=h$ provided $|\xi|$ is sufficiently large. Choose $h_{1}=(L-\lambda I) w^{*}$, as required. Hence the proof is completed.

Now we set $\bar{\iota}=\max \left\{a^{+}+b^{+}, a^{-}+b^{-}\right\}, \underline{\iota}=\min \left\{a^{+}-b^{+}, a^{-}-b^{-}\right\}, \quad \Omega_{+}=\{\lambda \in \mathbb{C} \mid \operatorname{Re} \lambda>\bar{\iota}\}$, $\Omega_{-}=\{\lambda \in \mathbb{C} \mid \operatorname{Re} \lambda<\underline{\iota}\}, \Xi=\{\lambda \in \mathbb{C} \mid \operatorname{Re} \lambda>\bar{\iota}\} \cup\left\{\lambda \in \mathbb{C}|| \operatorname{Im} z \mid>c^{2} \sqrt{\bar{\iota}-\operatorname{Re} z}+\left(b^{+} \wedge b^{-}\right)\right\}$.

Proposition 2.13. If $\lambda \in \Xi$ then $L-\lambda I$ is asymptotically hyperbolic when $d>0$. In case $d=0$, the same conclusion also holds if $\lambda \in \Omega_{+} \cup \Omega_{-}$.

Proof. It is sufficient to prove that $\Delta_{L_{ \pm}-\lambda}(i \eta) \neq 0$ for any $\eta \in \mathbb{R}$ provided $\operatorname{Re} \lambda>\bar{\iota}$. Note that $\Delta_{L_{ \pm}-\lambda}(i \eta)=0$ if and only if

$$
i c \eta+d \eta^{2}+\left(\lambda-a^{ \pm}\right)=b^{ \pm} \int_{\mathbb{R}} J(s) e^{-i \eta s} d s .
$$

First we note that

$$
d \eta^{2}+\operatorname{Re} \lambda-a^{ \pm}>b^{ \pm} \geq\left|b^{ \pm} \int_{\mathbb{R}} J(s) e^{-i \eta s} d s\right|, \quad \eta \in \mathbb{R}
$$

when $\operatorname{Re} \lambda>\bar{\iota}$, hence $\Delta_{L_{ \pm}-\lambda}(i \eta) \neq 0$ for all $\eta \in \mathbb{R}$, provided $\operatorname{Re} \lambda>\bar{\iota}$. Now, suppose $\operatorname{Re} \lambda \leq \bar{\iota}$, it is easy to see that

$$
c^{-2}\left(\operatorname{Im} z-b^{ \pm} \int_{\mathbb{R}} J(s) \sin (-\eta s) d s\right)^{2}+\operatorname{Re} z-a^{ \pm}>b^{ \pm} \int_{\mathbb{R}} J(s) \cos (\eta s) d s, \quad \eta \in \mathbb{R},
$$

whenever $|\operatorname{Im} z|>c^{2} \sqrt{\bar{\iota}-\operatorname{Re} z}+\left(b^{+} \wedge b^{-}\right)$. In case that $d=0$, we still have $\Delta_{L_{ \pm}-\lambda}(i \eta) \neq 0$ if $\operatorname{Re} \lambda>\bar{\iota}$. Moreover, $\operatorname{Re} \lambda<\underline{\iota}$ implies

$$
\operatorname{Re} \lambda-a^{ \pm}<-b^{ \pm} \leq \operatorname{Re}\left(b^{ \pm} \int_{\mathbb{R}} J(s) e^{-i \eta s} d s\right), \quad \eta \in \mathbb{R} .
$$

Therefore, the desired conclusion follows.

Proposition 2.14. Let $(c, U)$ be the solution to (1.1), then there exist positive constants $\nu$ and $C_{\nu}$ such that

$$
\left|U^{\prime}(\xi)\right| \leq C_{\nu} e^{\left(\lambda_{+}^{s}+\nu\right) \xi}, \quad \xi \geq 0
$$

and

$$
\left|U^{\prime}(\xi)\right| \leq C_{\nu} e^{\left(\lambda_{-}^{u}-\nu\right) \xi}, \quad \xi \leq 0,
$$

where $\lambda_{+}^{s}<0$ is the negative zero of $\Delta_{L_{+}}, \lambda_{-}^{u}>0$ is the positive zero of $\Delta_{L_{-}}$, and $\nu<\min \left\{-\lambda_{+}^{s}, \lambda_{-}^{u}\right\}$.

Proof. We shall retain the notations used in Lemma 2.8, while let $a(\xi)=f_{r}(U, J * U), b(\xi)=f_{s}(U, J * u)$, $a^{ \pm}=f_{r}( \pm 1, \pm 1)$, and $b^{ \pm}=f_{s}( \pm 1, \pm 1)$. Let $V$ stand for $U^{\prime}$. Then

$$
d V^{\prime \prime}+c V^{\prime}+f_{r}(U, J * U) V+f_{s}(U, J * U) J * V=0 .
$$

Namely, $(L V)(\xi)=0$. Due to Proposition 2.13 and (H4), $L$ is asymptotically hyperbolic. Note that the existence of $\lambda_{+}^{s}$ and $\lambda_{-}^{u}$ is guaranteed by Lemma 2.2. Let $L_{q^{ \pm}}$be the linear operators given in Lemma 2.8 and $G_{q^{ \pm}}$be the Green functions for $L_{q^{ \pm}}$. In view of the proofs of Proposition 2.4 and Proposition 2.7, there exist positive constants $\nu$ and $C_{1}$ such that $\left|G_{q^{+}}(\xi, \eta)\right| \leq C_{1} e^{\left(\lambda_{+}^{s}+\nu\right)|\xi-\eta|}$ and $\left|G_{q^{-}}(\xi, \eta)\right| \leq C_{1} e^{\left(\lambda_{-}^{u}-\nu\right)|\xi-\eta|}$, while $\nu<\min \left\{-\lambda_{+}^{s}, \lambda_{-}^{u}\right\}$. In particular, Lemma 2.8 shows that, for every $\xi \in \mathbb{R}$,

$$
V(\xi)=\int_{-\infty}^{\infty} G_{q^{+}}(\xi, \eta)\left[\left(L_{q}^{+}-L\right) V(\eta)\right] d \eta=\int_{-\infty}^{\tau} G_{q^{+}}(\xi, \eta)\left[\left(L_{q}-L\right) V(\eta)\right] d \eta,
$$


where we used the fact that $\left(L_{q}^{+}-L\right) V(\eta)=0$ for all $\eta \geq \tau$. Consequently, for any $\xi \geq \tau$, we have

$$
|V(\xi)| \leq C_{1} \int_{-\infty}^{\tau} e^{\left(\lambda_{+}^{s}+\nu\right)(\xi-\eta)}\left\|L_{q}^{+}-L\right\|\|V\|_{L^{\infty}} d \eta \leq \widetilde{C}_{1} e^{\left(\lambda_{+}^{s}+\nu\right) \xi} .
$$

Since $V$ is bounded on $\mathbb{R}$, it is possible to choose $C_{\nu}>0$ such that the desired estimate (2.25) holds for all $\xi \geq 0$. Analogously,

$$
\left|U^{\prime}(\xi)\right| \leq C_{\nu} e^{\left(\lambda_{+}^{u}-\nu\right) \xi}, \quad \xi \leq 0 .
$$

The proof is completed.

Now we are ready to state and prove the main result in this section

Theorem 2.15. Let $(c, U)$ be the solution to (1.1), then there exist positive constants $D_{1}$ and $D_{2}$ such that

$$
U(\xi)=1-D_{1} e^{\lambda_{+}^{s} \xi}[1+o(1)] \text { as } \xi \rightarrow \infty,
$$

and

$$
U(\xi)=-1+D_{2} e^{\lambda_{-}^{u}} \xi[1+o(1)] \text { as } \xi \rightarrow-\infty,
$$

where $\lambda_{+}^{s}<0$ and $\lambda_{-}^{u}>0$ are the roots of $\Delta_{L_{ \pm}}(z)$, respectively.

Proof. Consider

$$
M_{ \pm}(\xi) v=\left[f_{r}(U, J * U)-f_{r}( \pm 1, \pm 1)\right] v+\left[f_{s}(U, J * U)-f_{s}( \pm 1, \pm 1)\right] J * v .
$$

We first show that

$$
\left|M_{+}(\xi) V(\xi)\right| \leq C_{2} e^{2\left(\lambda_{+}^{s}+\nu\right) \xi}, \quad \xi \geq 0 ; \quad\left|M_{-}(\xi) V(\xi)\right| \leq C_{2} e^{2\left(\lambda_{-}^{u}-\nu\right) \xi}, \quad \xi \leq 0
$$

hold for some constant $C_{2}>0$. In fact, by the mean value theorem, we have

$$
\left|M_{ \pm}(\xi) V(\xi)\right| \leq \widetilde{K}[|U(\xi) \mp 1|+|J *(U \mp 1)(\xi)|][|V(\xi)|+|J * V(\xi)|]
$$

for some positive constant $\widetilde{K}$. It follows from Proposition 2.14 that

$$
|U(\xi)-1| \leq\left(\lambda_{+}^{s}+\nu\right)^{-1} C_{\nu} e^{\left(\lambda_{+}^{s}+\nu\right) \xi}, \quad \xi \geq 0 ; \quad \text { and }|U(\xi)+1| \leq\left(\lambda_{-}^{u}-\nu\right)^{-1} C_{\nu} e^{\left(\lambda_{-}^{u}-\nu\right) \xi}, \quad \xi \leq 0 .
$$

Hence, for any $\xi \geq 0$,

$$
\begin{aligned}
& |J *(U-1)(\xi)| \\
= & \left|\int_{\mathbb{R}} J(\eta)(U(\xi-\eta)-1) d \eta\right| \leq \int_{-\infty}^{\xi}+\int_{\xi}^{\infty} J(\eta)|U(\xi-\eta)-1| d \eta \\
\leq & \frac{C_{\nu} e^{\left(\lambda_{+}^{s}+\nu\right) \xi}}{\left(\lambda_{+}^{s}+\nu\right)} \int_{\mathbb{R}} J(\eta) e^{-\left(\lambda_{+}^{s}+\nu\right) \eta} d \eta+e^{\left(\lambda_{+}^{s}+\nu\right) \xi} \int_{\mathbb{R}} J(\eta) e^{-\left(\lambda_{+}^{s}+\nu\right) \eta}|U(\xi-\eta)-1| d \eta \\
\leq & C^{\prime} e^{\left(\lambda_{+}^{s}+\nu\right) \xi} .
\end{aligned}
$$

Similarly,

$$
|J *(U+1)(\xi)| \leq C e^{\left(\lambda_{-}^{u}-\nu\right) \xi}, \quad \xi \leq 0,
$$

and

$$
|J * V(\xi)| \leq\left\{\begin{array}{l}
C_{3} e^{\left(\lambda_{+}^{s}+\nu\right) \xi} \text { if } \xi \geq 0 \\
C_{3} e^{\left(\lambda_{-}^{u}-\nu\right) \xi} \text { if } \xi \leq 0
\end{array}\right.
$$

Therefore, (2.29) follows. 
Now set $h_{ \pm}(\xi)=-M_{ \pm}(\xi) U(\xi)$. As long as $\nu$ is sufficiently small, there exists $\iota>0$ such that $2\left(\lambda_{+}^{s}+\nu\right) \leq \lambda_{+}^{s}-\iota$ and $2\left(\lambda_{-}^{u}-\nu\right) \geq \lambda_{-}^{u}+\iota$. Therefore, we have

$$
\left|h_{+}(\xi)\right| \leq C_{2} e^{\left(\lambda_{+}^{s}-\iota\right) \xi} \text { for any } \xi \geq 0 ; \quad\left|h_{-}(\xi)\right| \leq C_{2} e^{\left(\lambda_{-}^{u}+\iota\right) \xi} \text { for any } \xi \leq 0 .
$$

In addition, due to the boundedness of $U \mp 1$ and $J *(U \mp 1)$, it is easy to see that

$$
\left|h_{+}(\xi)\right|=O\left(e^{\left(\lambda_{-}^{u}-\nu\right) \xi}\right) \quad \text { as } \xi \rightarrow-\infty ; \quad\left|h_{-}(\xi)\right|=O\left(e^{\left(\lambda_{+}^{s}+\nu\right) \xi}\right) \text { as } \xi \rightarrow \infty .
$$

Clearly, we have

$$
L_{ \pm} V=h_{ \pm}(\xi)
$$

In particular, when $d=0$,

$$
\left|V^{\prime}(\xi)\right| \leq\left|b^{ \pm}\right||J * V(\xi)|+\left|a^{ \pm}\right||V(\xi)|+\left|h_{ \pm}(\xi)\right| .
$$

We also observe that $h_{ \pm}$are differentiable and

$$
\left|h_{ \pm}^{\prime}(\xi)\right| \leq K^{\prime}\left[|V(\xi)|^{2}+|V(\xi)||J * V(\xi)|+|U(\xi) \mp 1|\left|V^{\prime}(\xi)\right|+|U(\xi) \mp 1|\left|J * V^{\prime}(\xi)\right|\right] .
$$

Therefore, it follows from (2.33) that

$$
\left|h_{+}^{\prime}(\xi)\right| \leq C_{4} e^{\left(\lambda_{+}^{s}-\iota\right) \xi} \text { for any } \xi \geq 0 ; \quad\left|h_{-}^{\prime}(\xi)\right| \leq C_{4} e^{\left(\lambda_{-}^{u}+\iota\right) \xi} \text { for any } \xi \leq 0 .
$$

Next, we show (2.27). Thanks to (2.30) and (2.31), $\widehat{h}_{+}(z)$ is analytic in the strip $0 \leq \operatorname{Im} z \leq 2 \epsilon-\lambda_{+}^{s}$, where $0<2 \epsilon<\iota$ and $\widehat{g}(z)=\frac{1}{2 \pi} \int_{\mathbb{R}} e^{-i z s} g(s) d s$. In case $d=0$, let $h_{+}^{\rho}(\xi)=h_{+}(\xi) e^{\rho \xi}$, where $\rho \in\left(0,2 \epsilon-\lambda_{+}^{s}\right)$. Due to $(2.34)$, for each $\rho \in\left(0,2 \epsilon-\lambda_{+}^{s}\right), h_{+}^{\rho} \in W^{1, p}(\mathbb{R})$ for any $p \geq 1$. Furthermore, we have

$$
|\eta|\left|\widehat{h}_{+}(\eta+i \rho)\right|=|\eta|\left|\widehat{h_{+}^{\rho}}(\eta)\right|=\left|i \eta \widehat{h_{+}^{\rho}}(\eta)\right|=\left|\widehat{\partial_{\eta} h_{+}^{\rho}}(\eta)\right| \leq\left\|\partial_{\eta} h_{+}^{\rho}\right\|_{L^{1}}, \quad \eta \in \mathbb{R} .
$$

Therefore, in the strip $0 \leq \operatorname{Im} z \leq 2 \epsilon-\lambda_{+}^{s}$,

$$
\left|\widehat{h}_{+}(z)\right|=O\left(|\operatorname{Re} z|^{-1}\right), \quad|\operatorname{Re} z| \rightarrow \infty .
$$

In the strip $|\operatorname{Re} z| \leq D$ with any fixed $D>0$, we have that $\Delta_{L_{+}}(z)=O(|\operatorname{Im} z|)\left(=O\left(|\operatorname{Im} z|^{2}\right)\right.$ if $d>0$ ) uniformly as $|\operatorname{Im} z| \rightarrow \infty$. Consequently, $\widehat{h}_{+}(-i z) \Delta_{L_{+}}^{-1}(z)=O(|\operatorname{Im} z|)^{-2}$ for any $z \in \mathbb{C}$ with $0<\operatorname{Re} z \leq \lambda_{+}^{s}-2 \epsilon$ and $\Delta_{L_{+}}(z) \neq 0$.

Since $L_{+}$is an isomorphism, $V$ is the unique solution to $L_{+} v=h_{+}$. By using Fourier transform and shifting the integrating path, when $\xi \geq 0$, we find

$$
\begin{aligned}
V & =\frac{1}{2 \pi} \int_{\mathbb{R}} \frac{e^{i \eta \xi} \widehat{h}_{+}(\eta)}{\Delta_{+}(i \eta)} d \eta=\frac{-i}{2 \pi} \int_{\mathbb{R}} \frac{e^{i \eta \xi \widehat{h}_{+}(-i(i \eta))}}{\Delta_{+}(i \eta)} d(i \eta) \\
& =\left.\sum \operatorname{Res} \frac{e^{z \xi} \widehat{h}_{+}(-i z)}{\Delta_{+}(z)}\right|_{\lambda_{+}^{s}-\epsilon \leq \operatorname{Re} z \leq 0}+\frac{-i}{2 \pi} \int_{\operatorname{Re} z=\lambda_{+}^{s}-\epsilon} \frac{e^{z \xi} \widehat{h}_{+}(-i z)}{\Delta_{L_{+}}(z)} d z .
\end{aligned}
$$

Here we choose $\epsilon$ such that $\Delta_{L_{+}}\left(\lambda_{+}^{s}-\epsilon+i \eta\right) \neq 0$ for any $\eta \in \mathbb{R}$. Clearly, The last integral converges absolutely.

Let $\Upsilon_{\lambda_{+}^{s}-\epsilon}=\left\{z \in \mathbb{C} \mid \Delta_{L^{+}}(z)=0, \lambda_{+}^{s}-\epsilon<\operatorname{Re} z<0\right\}$. Since $\widehat{h}(z)$ is analytic in the strip $0 \leq \operatorname{Im} z \leq$ $2 \epsilon-\lambda_{+}^{s}$, in the strip $\lambda_{+}^{s}-2 \epsilon \leq \operatorname{Re} z \leq 0, \widehat{h}(-i z) \Delta_{L_{+}}^{-1}(z)$ is meromorphic and only has poles which may occur at $z \in \Upsilon_{\lambda_{+}^{s}-\epsilon}$. We claim that $\widehat{h}(i z) \Delta_{L_{+}}^{-1}(z)$ has a simple pole at $z=\lambda_{+}^{s}$. Suppose this is not true. In virtue of Lemma 2.2, in the strip $\lambda_{+}^{s}-\epsilon<\operatorname{Re} z<0$, either all the poles of $\widehat{h}(-i z) \Delta_{L_{+}}^{-1}(z)$ occur at $z \in \Upsilon_{\lambda_{+}^{s}-\epsilon}$ with $\operatorname{Re} z<\lambda_{+}^{s}$ or $\widehat{h}(-i z) \Delta_{L_{+}}^{-1}(z)$ is analytic. For the latter, $V(\xi)=O\left(e^{\left(\lambda_{+}^{s}-\epsilon\right) \xi}\right)$, as $\xi \rightarrow \infty$. 
Certainly, $h_{+}(\xi)=O\left(e^{2\left(\lambda_{+}^{s}-\epsilon\right) \xi}\right)$ and $\widehat{h}(i z)$ is analytic in the strip $2 \lambda_{+}^{s}-\varkappa$ for some $0<\varkappa \leq 2 \epsilon$. Hence the path of integration can be shifted to the line $\operatorname{Re} z=2 \lambda_{+}^{s}-\varkappa$. Consequently, one of the following cases must occur.

Case I. The set $Z$ is not empty, where

$$
Z=\left\{\operatorname{Re} z \in \mathbb{R}^{-} \mid \Delta_{L_{+}}(z)=0, \widehat{h}(-i z) \text { is analytic at } z \text { and } \widehat{h}(-i z) \Delta_{L_{+}}^{-1}(z) \text { has poles at } z\right\} .
$$

Case II. $V(\xi)=O\left(e^{-b \xi}\right)$ for any $b \in \mathbb{R}^{+}$.

Next we show that both cases I and II are impossible. We start with case I. Let $\varrho=\sup Z$. Recall that $\lambda_{+}^{s}$ is the only real zero of $\Delta_{L_{+}}$in the half plane $\operatorname{Re} z \leq 0$. By Lemma 2.2, we may assume $\varrho \pm \mu_{m} i$ with $\mu_{m}>0(1 \leq m<\infty)$ are all the element of $Z$ with real part equal to $\varrho$. Suppose that $\widehat{h}(-i z) \Delta_{L_{+}}^{-1}(z)$ has a pole of order $l_{m}+1$ at $\varrho+\mu_{m} i$. Then

$$
\begin{aligned}
V(\xi) & =\sum \operatorname{Res}\left(e^{\xi z} \widehat{h}(i z) \Delta_{L_{+}}^{-1}(z)\right)_{\operatorname{Re} z=\varrho}+o\left(e^{\varrho \xi}\right) \\
& =\sum_{m} p_{l_{m}}(\xi) e^{\varrho \xi} \cos \left(\mu_{m} \xi+k_{m}\right)+o\left(e^{\varrho \xi}\right),
\end{aligned}
$$

where $p_{l_{m}}$ are real polynomials and $k_{m} \in \mathbb{R}$. Thus, $V(\xi)=\xi^{N} e^{\varrho \xi}\left(q(\xi)+O\left(\xi^{-1}\right)\right)$ as $\xi \rightarrow \infty$ for some $N>0$, where $q$ is a quasiperiodic function of mean value zero. According to Mallet-Paret [23],

$$
\liminf _{\xi \rightarrow \infty} q(\xi)<0<\limsup _{\xi \rightarrow \infty} q(\xi) .
$$

Consequently, $V\left(\xi_{1}\right)<0$ for some $\xi_{1}>0$. This contradicts the fact that $V(\xi)>0$ for any $\xi \in(-\infty, \infty)$. Therefore case I never occurs.

For case II, we define

$$
V_{b}=\int_{\mathbb{R}} V(\xi) e^{b \xi}, \quad b \in \mathbb{R}^{+}
$$

Note that

$$
\int_{\mathbb{R}} e^{b \xi} J * V(\xi) d \xi=\int_{\mathbb{R}} e^{b \eta} V(\eta) \int_{\mathbb{R}} e^{b(\xi-\eta)} J(\xi-\eta) d \xi d \eta=V_{b} \int_{\mathbb{R}} e^{b \xi} J(\xi) d \xi
$$

Let

$$
\underline{a}=\min _{[-1,1] \times[-1,1]} f_{r}(r, s), \quad \underline{b}=\min _{[-1,1] \times[-1,1]} f_{s}(r, s) .
$$

Due to (H2) and (H3), $\underline{a}>-\infty$ and $\underline{b}>0$. Consequently,

$$
c V^{\prime}-d V^{\prime \prime} \geq \underline{a} V+\underline{b} J * V
$$

Multiplying each side of (2.35) by $e^{b \xi}$ and integrating by part yield

$$
\left(-c b-d b^{2}-\int_{\mathbb{R}} J(\xi) e^{b \xi} d \xi\right) V_{b} \geq \underline{a} V_{b},
$$

thus

$$
-c b-d b^{2}-\underline{b} \int_{\mathbb{R}} J(\xi) e^{b \xi} d \xi \geq \underline{a} .
$$

Since $-c b-d b^{2}-\int_{\mathbb{R}} J(\xi) e^{b \xi} d \xi \rightarrow-\infty$, as $b \rightarrow \infty$, we arrive at a contradiction. This implies that case II cannot occur. Therefore, $e^{z \xi} \widehat{h}(-i z) \Delta_{L_{+}}^{-1}(z)$ has a simple pole at $z=\lambda_{+}^{s}$, and

$$
\begin{aligned}
V(\xi) & =\frac{e^{\lambda_{+}^{s} \xi \widehat{h}_{+}\left(-i \lambda_{+}^{s}\right)}}{\Delta_{L_{+}}^{\prime}\left(\lambda_{+}^{s}\right)}+\frac{1}{2 \pi} \int_{\operatorname{Re} z=\lambda_{+}^{s}-\epsilon} \frac{e^{z \xi \widehat{h}_{+}}(-i z)}{\Delta_{L_{+}}(z)} d z \\
& =\frac{e^{\lambda_{+}^{s} \xi} \int_{\mathbb{R}} h(\eta) e^{-\lambda_{+}^{s} \eta} d \eta}{\int_{\mathbb{R}} \eta J(\eta) e^{-\lambda_{+}^{s} \eta} d \eta-c}+\frac{e^{\left(\lambda_{+}^{s}-\epsilon\right) \xi}}{2 \pi} \int_{\mathbb{R}} \frac{e^{i \xi s} \widehat{h}_{+}\left(\eta+i\left(\epsilon-\lambda_{+}^{s}\right)\right)}{\Delta_{L_{+}}\left(\lambda_{+}^{s}-\epsilon+i \eta\right)} d \eta .
\end{aligned}
$$


Now let

$$
\gamma^{+}=\frac{\int_{\mathbb{R}} h(\eta) e^{-\lambda_{+}^{s} \eta} d \eta}{\int_{\mathbb{R}} \eta J(\eta) e^{-\lambda_{+}^{s} \eta} d \eta-c}, \quad V^{+}(\xi)=\frac{e^{\left(\lambda_{+}^{s}-\epsilon\right) \xi}}{2 \pi} \int_{\mathbb{R}} \frac{e^{i \xi s} \widehat{h}_{+}\left(\eta+i\left(\epsilon-\lambda_{+}^{s}\right)\right)}{\Delta_{L_{+}}\left(\lambda_{+}^{s}-\epsilon+i \eta\right)} d \eta .
$$

Clearly, $V^{+}(\xi)=o\left(e^{\lambda_{+}^{s} \xi}\right)$ as $\xi \rightarrow+\infty$. The positivity of $V$ forces that $\gamma^{+}>0$. Thus,

$$
U^{\prime}(\xi)=\gamma^{+} e^{\lambda_{+}^{s} \xi}+o\left(e^{\lambda_{+}^{s} \xi}\right), \quad \xi \rightarrow+\infty .
$$

By considering the equation $L_{-} V=h_{-}$and arguing analogously, we may find

$$
U^{\prime}(\xi)=\gamma^{-} e^{\lambda_{-}^{u} \xi}+o\left(e^{\lambda_{-}^{u} \xi}\right), \quad \xi \rightarrow-\infty
$$

for some constant $\gamma^{-}>0$. With the boundary conditions $U( \pm \infty)= \pm 1$, we are readily to obtain the desired conclusions.

The uniqueness of a monotone traveling wave $U$ for the nonlocal Allen-Cahn equation (1.1) with $d=0$ was established in Bates et al. [11] and late for the general equation (1.1) in Chen [15]. In those works, the uniqueness of speed and profile of traveling wave solution $U$ are obtained by means of a comparison principle and sub-and super-solution techniques. Here we provided a technically different and simplified proof for the uniqueness of $U$.

Corollary 2.16. Assume that (H1)-(H5) are satisfied. Then there exists a unique $c^{*} \in \mathbb{R}$ such that equation (1.1) possesses a solution satisfying (2.1) if and only if $c=c^{*}$ and the traveling wave solution $U$ is unique up to translation of $\xi$.

Proof. We shall argue by contradiction. Suppose that there exist $\left(c_{i}, U_{i}\right)$ satisfying (1.1) with $c_{1}<c_{2}$ , $i=1,2$. We may assume that one of these solutions has speed $c^{*}$. By Theorem 2.15, both solutions satisfy

$$
U_{i}(\xi)= \begin{cases}-1+n_{i} e^{e_{i}^{u} \xi}+o\left(e^{\varrho_{i}^{u} \xi}\right), & \xi \rightarrow-\infty \\ 1-\widetilde{n}_{i} e^{e_{i}^{s} \xi}+o\left(e^{\varrho_{i}^{s} \xi}\right), \quad \xi \rightarrow \infty\end{cases}
$$

for some $n_{i}, \widetilde{n}_{i}>0$ and $\varrho_{i}^{u}>0, \varrho_{i}^{s}<0$. Furthermore, $\varrho_{i}^{u}$ and $\varrho_{i}^{s}$ satisfy

$$
\begin{gathered}
c_{i} \varrho_{i}^{s}-d\left(\varrho_{i}^{s}\right)^{2}-a^{+}-b^{+} \int_{\mathbb{R}} J(s) e^{-\varrho_{i}^{s} s} d s=0, \\
c_{i} \varrho_{i}^{u}-d\left(\varrho_{i}^{u}\right)^{2}-a^{-}-b^{-} \int_{\mathbb{R}} J(s) e^{-\varrho_{i}^{u} s} d s=0 .
\end{gathered}
$$

In view of the proof of Lemma 2.2, it is easy to see that

$$
\varrho_{1}^{s}<\varrho_{2}^{s}<0, \quad 0<\varrho_{1}^{u}<\varrho_{2}^{u} .
$$

Thus, $U_{2}(\xi)<U_{1}(\xi)$ for all sufficiently large $|\xi|$. This together with the monotonicity of $U_{i}$ justify that we can choose $\tau \in \mathbb{R}$ and replace $U_{2}(\xi)$ by $U_{2}(\xi+\tau)$ such that $U_{2}(\xi) \leq U_{1}(\xi)$ for all $\xi \in \mathbb{R}$ and $U_{2}\left(\xi_{0}\right)=U_{1}\left(\xi_{0}\right)$ for some $\xi_{0}$. Consequently, $U_{2}^{\prime}\left(\xi_{0}\right)=U_{1}^{\prime}\left(\xi_{0}\right)$ and $U_{2}^{\prime \prime}\left(\xi_{0}\right) \leq U_{1}^{\prime \prime}\left(\xi_{0}\right)$. Moreover, (H3) and the fact that $J * U_{1}\left(\xi_{0}\right)>J * U_{2}\left(\xi_{0}\right)$ imply that $f\left(U_{1}\left(\xi_{0}\right), J * U_{1}\left(\xi_{0}\right)\right)>f\left(U_{2}\left(\xi_{0}\right), J * U_{2}\left(\xi_{0}\right)\right)$. By plugging these relations into (1.1), we find

$$
0=d U_{1}^{\prime \prime}\left(\xi_{0}\right)-c_{1} U_{1}^{\prime}\left(\xi_{0}\right)+f\left(U_{1}\left(\xi^{*}\right), J * U_{1}\left(\xi_{0}\right)\right)>d U_{2}^{\prime \prime}\left(\xi_{0}\right)-c_{2} U_{2}^{\prime}\left(\xi_{0}\right)+f\left(U_{2}\left(\xi_{0}\right), J * U_{2}\left(\xi_{0}\right)\right)=0 .
$$

The contradiction completes the proof. 


\section{Spectral analysis of traveling waves}

In this section, we again let $a(\xi)=f_{r}(U, J * U), b(\xi)=f_{s}(U, J * u), a^{ \pm}=f_{r}( \pm 1, \pm 1)$, and $b^{ \pm}=f_{s}( \pm 1, \pm 1)$. Recall that

$$
(L u)(\xi):=d u^{\prime \prime}(\xi)-c u^{\prime}(\xi)+f_{r}(U, J * U) u(\xi)+f_{s}(U, J * U)(J * u)(\xi)
$$

and

$$
\left(L_{ \pm} u\right)(\xi):=d u^{\prime \prime}(\xi)-c u^{\prime}(\xi)+a^{ \pm} u(\xi)+b^{ \pm}(J * u)(\xi) .
$$

We now study the spectrum of the operator $L$. Throughout the rest of the paper, we let $X:=$ $L^{p}(\mathbb{R}, \mathbb{C}), 1 \leq p \leq \infty$. $\operatorname{Re} X$ is considered as an ordered Banach space with a positive cone $X_{+}$, where $\operatorname{Re} X=\{\operatorname{Re} u \mid u \in X\}$ and $X_{+}=\{w \in \operatorname{Re} X \mid w \geq 0\}$. It is well known that $X_{+}$is generating and normal (see Amann [1] for more details). For $\varphi \in \operatorname{Re} X$, we write $\varphi \gtrless 0$ if $\varphi \in X_{+}$and $\varphi \neq 0, \varphi \gg 0$ if $\varphi(\xi)>0$ for each $\xi \in \mathbb{R}$. An operator $A: X \rightarrow X$ is called positive if $A X_{+} \subseteq X_{+}$.

Definition 3.1. An operator $A$ is said to be resolvent positive if the resolvent set $\rho(A)$ of $A$ contains an interval $(\alpha, \infty)$ and $(\lambda I-A)^{-1}$ is positive for sufficiently large $\lambda \in \rho(A) \cap \mathbb{R}$.

In sequel, we follow Henry [19] to define the normal points and the essential spectrum of an operator $A$ on a Banach space. Namely, a normal point of $A$ is a complex number in the resolvent set $\rho(A)$ or an isolated eigenvalue of $A$ with finite multiplicity. The complement of the set of normal points is called the essential spectrum of $A$, denoted by $\sigma_{\text {ess }}(A)$. We denote the spectral bound of an operator $A$ by

$$
\mathfrak{s}(A)=\sup \{\operatorname{Re} \lambda: \lambda \in \sigma(A)\} .
$$

We also let $\bar{\iota}=\max \left\{a^{+}+b^{+}, a^{-}+b^{-}\right\}$and $\underline{\iota}=\min \left\{a^{+}-b^{+}, a^{-}-b^{-}\right\}$, where $a^{ \pm}=f_{r}( \pm 1, \pm 1), b^{ \pm}=$ $f_{s}( \pm 1, \pm 1)$.

Theorem 3.2. Consider the linear operator $L: L^{p} \rightarrow L^{p}$ defined by (3.1), which corresponds to the variational equation of (1.1) at $U$, that is,

$$
(L u)(\xi)=d u^{\prime \prime}(\xi)-c u^{\prime}(\xi)+f_{r}(U, J * U) u(\xi)+f_{s}(U, J * U)(J * u)(\xi)
$$

and its formally adjoint operator $L^{*}: L^{q} \rightarrow L^{q}$ defined by

$$
\left(L^{*} u\right)(\xi)=d u^{\prime \prime}(\xi)+c u^{\prime}(\xi)+f_{r}(U, J * U) u(\xi)+\left(J * f_{s}(U, J * U) u\right)(\xi),
$$

where $1 \leq p \leq \infty, \frac{1}{p}+\frac{1}{q}=1$. $q=\infty$ if $p=1$, and $q=1$ if $p=\infty$. Then

(I) Case $d=0$.

(i) Let $\Omega_{+}=\{\lambda \in \mathbb{C} \mid \operatorname{Re} \lambda>\bar{\iota}\}$ and $\Omega_{-}=\{\lambda \in \mathbb{C} \mid \operatorname{Re} \lambda<\underline{\iota}\}$. Then $\lambda$ is an isolated eigenvalue with a finite algebraic multiplicity if $\lambda \in\left(\Omega_{+} \cup \Omega_{-}\right) \cap \sigma(L)$. Furthermore, suppose $\psi$ is an eigenfunction corresponding to $\lambda$, then

$$
|\psi(\xi)| \leq C_{\lambda} e^{-\mu|\xi|}, \quad \xi \in \mathbb{R}
$$

for some positive constants $C_{\lambda}$ and $\mu$;

(ii) $\sigma_{\mathrm{ess}}(L) \subseteq\{\lambda \in \mathbb{C} \mid \underline{\iota} \leq \operatorname{Re} \lambda \leq \bar{\iota}\}$;

(iii) $\mathfrak{s}(L)=\mathfrak{s}\left(L^{*}\right)=0$ and 0 is a simple eigenvalue for both $L$ and $L^{*}$;

(iv) $\operatorname{dim} \mathcal{N}(L)=\operatorname{dim} \mathcal{N}\left(L^{*}\right)=\operatorname{codim} \mathcal{R}(L)=\operatorname{codim} \mathcal{R}\left(L^{*}\right)=1$. Moreover, $L^{*}$ has a positive eigenfunction $\Psi$ corresponding to the eigenvalue 0 , and

$$
\mathcal{R}(L)=\left\{h \in L^{p} \mid \int_{\mathbb{R}} \Psi(\xi) h(\xi) d \xi=0\right\}
$$

(v) There exist $\varpi>0$ such that the set $\{\lambda \in \mathbb{C} \mid \operatorname{Re} \lambda<-\varpi$, or $\operatorname{Re} \lambda \geq 0$ and $\lambda \neq 0\} \subset \rho(L)$, where $\rho(L)$ denotes the resolvent set of $L$. 
(II) Case $d>0$.

(i) Let $\Xi=\left\{\lambda \in \mathbb{C}|| \operatorname{Im} \lambda \mid>\sqrt{\bar{\iota}-\operatorname{Re} \lambda}+\left(b^{-} \wedge b^{+}\right), \operatorname{Re} \lambda \leq \bar{\iota}\right\} \cup\{\operatorname{Re} \lambda>\bar{\iota}\}$. Suppose that $\lambda \in$ $\Xi \cap \sigma(L)$, then $\lambda$ is an isolated eigenvalue with a finite algebraic multiplicity. Furthermore, if $\psi$ is an eigenfunction corresponding to $\lambda$ then

$$
|\psi(\xi)| \leq C_{\lambda} e^{-\mu|\xi|}, \quad \xi \in \mathbb{R},
$$

where $C_{\lambda}$ and $\mu$ are positive constants;

(ii) $\sigma_{\mathrm{ess}}(L) \subseteq \mathbb{C} \backslash \Xi$;

(iii)-(iv) The assertions (iii) and (iv) stated in case I above remain true;

(v) The set $\{\lambda \in \mathbb{C} \mid \operatorname{Re} \lambda \geq 0$ and $\lambda \neq 0\} \subset \rho(L)$.

Proof. We shall first prove that $L$ is resolvent positive. The proof for $L^{*}$ is same. Let $\widetilde{\lambda}>0$ be sufficiently large and write $\tilde{\lambda}=\lambda^{*}-\underline{\lambda}$ such that $\max _{0 \leq U \leq 1}\left|f_{r}(U, J * U)\right| \leq \underline{\lambda}<\infty$. Then we have $(\widetilde{\lambda} I-L)=\left(\lambda^{*} I+c \partial-d \partial^{2}\right)-(\underline{\lambda} I+P)$, where $(P v)(\xi):=f_{r}(U, J * U) v(\xi)+f_{s}(U, J * U)(J * v)$ and $\partial$ denotes differentiation. According to Miklavcic [24] (see section 1.6), As long as $\lambda^{*}$ is sufficiently large, $\left(\lambda^{*} I+c \partial-d \partial^{2}\right)$ is invertible and positive. In particular, $\left\|\left(\lambda^{*} I+c \partial-d \partial^{2}\right)^{-1}\right\| \leq\left|\frac{k}{\lambda^{*}}\right|$ for some $k>0$ provided $d>0$, and $\left\|\left(\lambda^{*} I+c \partial\right)^{-1}\right\| \leq\left|\frac{c}{\lambda^{*}}\right|$. Since $P$ is a bounded operator, $(\tilde{\lambda} I-L)$ is invertible provided $\widetilde{\lambda}$ is sufficiently large. Therefore, we have

$$
\begin{aligned}
(\widetilde{\lambda} I-L)^{-1} & =\left[\left(\lambda^{*} I+c \partial-d \partial^{2}\right)-(\underline{\lambda} I+P)\right]^{-1} \\
& =\left(\lambda^{*} I+c \partial-d \partial^{2}\right)^{-1}\left[I-(\underline{\lambda} I+P)\left(\lambda^{*} I+c \partial-d \partial^{2}\right)^{-1}\right] \\
& =\left(\lambda^{*} I+c \partial-d \partial^{2}\right)^{-1} \sum_{j=0}^{\infty}\left[(\underline{\lambda} I+P)\left(\lambda^{*} I+c \partial-d \partial^{2}\right)^{-1}\right]^{j} .
\end{aligned}
$$

Note that $(\underline{\lambda} I+P)$ is positive. So the above Neumann series is a sum of positive operators and hence is positive. Clearly, for any $\lambda>\widetilde{\lambda},(\lambda I-L)$ is invertible and positive. Hence $L$ is resolvent positive.

Next, we prove the statements (i)-(v) for the case that $d=0$. The proof for the case that $d>0$ follows the same lines and shall be omitted.

By Miklavcic [24] again, there exists $\varpi>0$ sufficiently large such that $(\lambda I+c \partial)$ is invertible and $\left\|(\lambda I+c \partial)^{-1}\right\| \leq\left|\frac{c}{\lambda}\right|$ whenever $\lambda \leq-\varpi$. With the same reasoning used previously, we see that $(\lambda I-L)$ is invertible provided $\lambda \leq-\varpi$. This proves (v).

Notice that both $\Omega_{+}$and $\Omega_{-}$are connected open subsets of $\mathbb{C}$. Due to Proposition 2.13 and Lemma 2.12 , both $(L-\lambda I)$ and $\left(L^{*}-\lambda I\right)$ are semi-Fredholm operators whenever $\lambda \in \Omega_{-} \cup \Omega_{+}$. Since $\rho(L) \cap \Omega_{+} \neq \emptyset$ and $\rho(L) \cap \Omega_{-} \neq \emptyset$, according the first paragraph on p.243 of Kato [18], the followings hold true:

(a1) $(L-\lambda I)$ is Fredholm of index zero if $\lambda \in \Omega_{-} \cup \Omega_{+}$.

(a2) Suppose that $\lambda \in \sigma(L) \cap\left(\Omega_{+} \cup \Omega_{-}\right)$, then $\lambda$ is an isolated eigenvalue with finite algebraic multiplicity.

Furthermore, Lemma 2.8 implies (3.3). Therefore (i) is completed. As a consequence of (i), (ii) is true. Next, we show (iii) and (iv). Analogously, (a1) and (a2) remain valid for $L^{*}$. Notice that $0 \in \sigma(L)$, so $\mathfrak{s}(L) \geq 0>-\infty$. By Thieme [28], the resolvent positivity yields that $\mathfrak{s}(L) \in \sigma(L)$. In particular, $\mathfrak{s}(L) \in \sigma(L) \cap \Omega_{+}$since $\bar{\iota}<0$. Therefore, (a1) and (a2) imply that $\operatorname{Ind}(L-\mathfrak{s}(L) I)=0$ and $\mathfrak{s}(L)$ is an isolated eigenvalue with finite algebraic multiplicity. It follows from Lemma 2.12 that

$$
\operatorname{codim} \mathcal{R}\left(L^{*}-\mathfrak{s}(L) I\right)=\operatorname{codim} \mathcal{R}(L-\mathfrak{s}(L) I)^{*} \geq \operatorname{dim} \mathcal{N}(L-\mathfrak{s}(L) I)>0 .
$$

Consequently, $\mathfrak{s}(L) \in \sigma\left(L^{*}\right)$. By the resolvent positivity of $L^{*}$, we infer that $\mathfrak{s}\left(L^{*}\right) \in \sigma\left(L^{*}\right)$ and $\mathfrak{s}\left(L^{*}\right) \geq$ $\mathfrak{s}(L) \geq 0$. Moreover, $\mathfrak{s}\left(L^{*}\right)$ has a positive eigenfunction $\Psi$. Suppose that $\mathfrak{s}\left(L^{*}\right)>0$. Observe that $U^{\prime}(\xi)$ is an eigenvalue of $L$ corresponding to eigenvalue 0 and hence $\mathfrak{s}\left(L^{*}\right) U^{\prime}(\xi) \in \mathcal{R}\left(L-\mathfrak{s}\left(L^{*}\right) I\right)$. Since $\left(L-\mathfrak{s}\left(L^{*}\right) I\right)^{*}=L^{*}-\mathfrak{s}\left(L^{*}\right) I$, it follows from Lemma 2.12 that

$$
\mathfrak{s}\left(L^{*}\right) \int_{\mathbb{R}} \Psi(\xi) U^{\prime}(\xi) d \xi=0,
$$


which is impossible since $U^{\prime} \gg 0$. Thus $\mathfrak{s}\left(L^{*}\right)=\mathfrak{s}(L)=0$. We now prove the simplicity of eigenvalue 0 , without loss of generality, we assume that $c>0$. We first show that $\mathcal{N}(L)=\operatorname{span}\left\{U^{\prime}\right\}$. Suppose this not true, then there is an eigenfunction $\psi$ associated with eigenvalue 0 such that $\psi \neq t U^{\prime}$ for all $t \in \mathbb{R}$. Obviously, $\psi \in W^{2, p}(\mathbb{R})$. In view of Theorem $2.15,|\psi(\xi)|=O\left(e^{\lambda_{+}^{s} \xi}\right)$ as $\xi \rightarrow \infty$, and $|\psi(\xi)|=O\left(e^{\lambda_{-}^{u} \xi}\right)$ as $\xi \rightarrow-\infty$. Due to the positivity of $U^{\prime}$, there exist $t$ such that $t U^{\prime}+\psi \geq 0$. Let $\bar{t}=\inf \left\{t \in \mathbb{R}: t U^{\prime}+\psi \geq 0\right\}$. Obviously, $\bar{t} U^{\prime}+\psi \neq 0$. Set $\bar{w}=\bar{t} U^{\prime}+\psi$ and $\Sigma=\{\xi \in \mathbb{R} \mid \bar{w}(\xi)=0\}$. Note that $\Sigma$ is not empty by our assumption. Furthermore, $\Sigma$ is a close set and $\Sigma \backslash \operatorname{int} \Sigma \neq \emptyset$. Let $\xi_{0} \in \Sigma \backslash \operatorname{int} \Sigma$. Certainly, for each $\varepsilon>0$, there is a point $\xi_{\varepsilon} \in\left(\xi_{0}-\frac{1}{2} \varepsilon, \xi_{0}+\frac{1}{2} \varepsilon\right)$ such that $\bar{w}\left(\xi_{\varepsilon}\right)>0$. Since, for any $\gamma>\max _{0 \leq U \leq 1}\left|f_{r}(U, J * U)\right|$, $c \bar{w}^{\prime}+\gamma \bar{w}=(P \bar{w}+\gamma \bar{w}) \gtrless 0$, simple calculation shows that

$$
\bar{w}(\xi)=\int_{-\infty}^{\xi} e^{-\frac{\gamma}{c}(\xi-\eta)}(P+\gamma I) \bar{w}(\eta) d \eta, \quad \xi \in \mathbb{R} .
$$

Clearly, $\bar{w}(\xi)>0$ for any $\xi \geq \xi_{0}+\varepsilon$. Thanks to (H1), there exist $a$ and $b$ with $b>a>0$ such that $(-b,-a) \cup(a, b) \subseteq \operatorname{supp} J$. Since $\varepsilon$ can be chosen sufficiently small such that $\varepsilon<a$, we find that $\operatorname{supp} J\left(\xi_{0}-\cdot\right) \cap \operatorname{supp} \bar{w}(\cdot)$ contains a nonempty open interval. Hence $J * \bar{w}\left(\xi_{0}\right)>0$. On the other hand, $\bar{w}^{\prime}\left(\xi_{0}\right)=0$ implies that

$$
0 \leq f_{s}(U, J * U)(J * \bar{w})\left(\xi_{0}\right) \leq(L \bar{w})\left(\xi_{0}\right)=0 .
$$

(H3) forces that $J * \bar{w}\left(\xi_{0}\right)=0$, thus we reach a contradiction. The contradiction leads to the desired conclusion that $\mathcal{N}(L)=\operatorname{span}\left\{U^{\prime}\right\}$. As mentioned early, we can similarly show that $\mathcal{N}(L)=\operatorname{span}\left\{U^{\prime}\right\}$ for the case that $d>0$. However, the proof is much simpler. Indeed, we have $c \bar{w}^{\prime}-d \bar{w}^{\prime \prime}+\gamma \bar{w}=(P \bar{w}+\gamma \bar{w}) \gtrless 0$, where $\gamma$ is the constant same as one defined above. Then

$$
\bar{w}(\xi)=\int_{-\infty}^{\xi} e^{\mu_{-}}(P+\gamma I) \bar{w}(\eta) d \eta+\int_{\xi}^{\infty} e^{\mu_{+}}(P+\gamma I) \bar{w}(\eta) d \eta, \quad \xi \in \mathbb{R}
$$

where $\mu_{ \pm}=\left[c \pm \sqrt{c^{2}+4 d \gamma}\right](2 d)^{-1}$. Thus, $\bar{w}=\bar{t} U^{\prime}+\psi \gg 0$, which violates the definition of $\bar{t}$, and the contradiction yields the conclusion we need. Next, we show that $\mathcal{N}(L)^{2}=\mathcal{N}(L)$ by contradiction. Let $L \Phi=t_{1} U^{\prime}$ for some $\Phi \in L^{p}$ and $t_{1} \in \mathbb{R}$, that is, $t_{1} U^{\prime} \in \mathcal{R}(L)$. Therefore, $t_{1} \int_{\mathbb{R}} \Psi(\eta) U^{\prime}(\eta) d \eta=0$, which is a contradiction. With the same reasoning, we can show that $\mathcal{N}\left(L^{*}\right)=\operatorname{span}\{\Psi\}$ and 0 is also a simple eigenvalue of $L^{*}$. Thus, we proved that (iii) and $\operatorname{dim} \mathcal{N}(L)=\operatorname{dim} \mathcal{N}\left(L^{*}\right)=\operatorname{codim} \mathcal{R}(L)=\operatorname{codim} \mathcal{R}\left(L^{*}\right)=$ 1. Note that (3.4) is ensured by Lemma 2.12 if $L$ is considered in $L^{p}$ with $1 \leq p<\infty$. In case that $p=\infty, \mathcal{R}(L) \subseteq\left\{h \in L^{\infty} \mid \int h \Psi=0\right\}$ implies (3.4). Hence (iv) is completed. Certainly, $\lambda \in \rho(L)$ for any $\lambda \in \mathbb{C}$ with $\operatorname{Re} \lambda>0$. Moreover, by using the arguments similar to those in Bates and Chen [10] (see p. 124 , also refer to Volpert et al. [29]), we can show that $L-i \eta$ is injective for any $\eta \in \mathbb{R}$. On the other hand, for each $\eta \in \mathbb{R}, L-i \eta$ is Fredholm of index zero. Hence $i \eta \in \rho(L)$ for any $\eta \in \mathbb{R}$ and (v) is proved.

Acknowledgements. Research was partially supported by NSF grant DMS-1412454.

\section{References}

[1] H. Amann, Fixed point equations and nonlinea eigenvalue problems in ordered Banach spaces, SIAM Rev. 18 (1976), 620-709.

[2] F. Andreu, J. M. Mazón, J. D. Rossi, J. Toledo, Nonlocal Diffusion Problems, Math. Surveys Monogr., Amer. Math. Soc., Providence, RI, 2010.

[3] N. Apreutesei, A. Ducrot and V. Volpert, Competition of species with intra-specific competition, Math. Model. Nat. Phenom. 3 (2008), 1-27.

[4] N. Apreutesei, N. Bessonov, V. Volpert and V. Vougalter, Spatial structures and generalized travelling waves for an integro-differential equation, Discrete Contin. Dynam. Syst. Ser. B. 13 (2010), 537-557.

[5] N. Apreutesei and V. Volpert, Properness and topological degree for nonlocal reaction-diffusion operators, Abstract Appl. Anal. (2011), 1-21. 
[6] N. Apreutesei and V. Volpert, Existence of travelling waves for a class of integro-differential equations from population dynamics, Intl. Electron. J. Pure Appl. Math. 5 (2012), 53-67.

[7] N. Apreutesei and V. Volpert, Properness and topological degree for nonlocal integro-differential systems, Topol. Methods Nonlinear Anal. 43 (2014), 215-229.

[8] D. G. Aronson, The asymptotic speed of propagation of a simple epidemic, in "Nonlinear Diffusion", eds. by W. E. Fitzgibbon and H. F. Walker, Research Notes in Math. 14, Pitman, London, 1977, pp. 1-23.

[9] P. W. Bates and A. Chmaj, An integrodifferential model for phase transitions: Stationary solution in higher space dimensions, J. Stat. Phys. 95 (1999), 1119-1139.

[10] P. W. Bates and F. Chen, Spectral analysis of traveling waves for nonlocal evolution equations, SIAM J. Math. Anal. 38 (2006), 116-126.

[11] P. W. Bates, P.C. Fife, X. Ren and X. Wang, Traveling waves in a nonlocal model of phase transitions, Arch. Rat. Mech. Anal. 138 (1997), 105-136.

[12] P. W. Bates, J. Han and G. Zhao, On a nonlocal phase-field system, Nonlinear Anal. 64 (2006), 2251-2278.

[13] P. W. Bates and G. Zhao, Existence, uniqueness and stability of stationary solution to a nonlocal evolution equation arising in population dispersal, J. Math. Anal. Appl. 332 (2007), 428-440.

[14] H. Brezis, Functional Analysis, Sobolev Spaces and Partial Differential Equations, Springer, New York, 2011.

[15] X. Chen, Existence, Uniqueness and asymptotic stability of traveling waves in nonlocal evolution equations, Adv. Differential Equations 2 (1997), 125-160.

[16] Z. Chen, B. Ermentrout and B. Mcleod, Traveling fronts for a class of non-local convolution differential equatons, Appl. Anal. 64 (1997), 235-253.

[17] A. De Masi, E. Orlandi, E. Presutti and L. Triolo, Stability of the interface in a model of phase separation, Proc. Roy. Soc. Edinburgh 124A (1994), 1013-1022.

[18] T. Kato, Perturbation Theory for Linear Operators, Springer Verlag, New York, 1976.

[19] D. Henry, Geometric Theory of Semilinear Parabolic Equations, Springer-Verlag, Berlin, 1981.

[20] W. Huang, Uniqueness of the bistable traveling wave for mutualist spaceies, J. Dynam. Differential Equations $\mathbf{1 3}$ (2001),147-183.

[21] H. J. Hupkes and S. M. Verduyn Lunel, Analysis of Newton's method to compute travelling waves in discrete media, J. Dynam. Differential Equations 17 (2005), 523-572.

[22] J. Mallet-Paret, The Fredholm alternative for functional differential equation of mixed type, J. Dynam. Differential Equations 11 (1999), 1-48.

[23] J. Mallet-Paret, The global structure of traveling waves in spatially discrete dynamical systems, J. Dynam. Differential Equations 11(1999), 49-127.

[24] M. Miklavcic, Applied Functional Analysis and Partial Differential Equations, World Scientific, Singapore, 1998.

[25] A. Pazy, Asymptotic expansions of solutions of ordinary differential equations in Hilbert space, Arch. Rat. Mech. Anal. 24 (1967), 105-136.

[26] S. Ruan, Spatial-temporal dynamics in nonlocal epidemiological models, in "Mathematics for Life Science and Medicine", Vol. 2, Y. Takeuchi, K. Sato and Y. Iwasa (eds.), Springer-Verlag, New York, 2007, pp. 97-122.

[27] K. H. Schumacher, Traveling front solutions for integro-differential equation I, J. Reine Angew. Math. 316 (1980), 54-70.

[28] H. R. Thieme, Remarks on resolvent positive operators and their perturbation, Discrete Contin. Dynam. Syst. 4 (1998), 73-90.

[29] A. I. Volpert, Vi. A. Volpert and Vl. A. Volpert, Traveling Wave Solutions of Parabolic Systems, Translated from Russian by J. F. Heyda, Transl. Math. Monogr., Vol. 140, Amer. Math. Soc., Providence, 1994.

[30] H. F. Weinberger, Long-time behavior of a class of biological models, SIAM J. Math. Anal. 13 (1982),353-396.

[31] G. Zhao, Multidimensional periodic traveling waves in infinite cylinders, Discrete Contin. Dynam. Syst. 24 (2009), 1025-1045.

[32] G. Zhao and S. Ruan, Existence, uniqueness and asymptotic stability of time periodic traveling waves for a periodic Lotka-Volterra competition system with diffusion, J. Math. Pures Appl. 95 (2011), 627-671.

[33] G. Zhao and S. Ruan, Time periodic traveling wave solutions for periodic advection-reaction-diffusion systems, J. Differential Equations 257 (2014), 1078-1147. 